\title{
Spatial Domain Suppression of Co-Channel Interference: A Reflection Amplifiers Surface (RAS) Approach
}

This paper was downloaded from TechRxiv (https://www.techrxiv.org).

\section{LICENSE}

CC BY 4.0

SUBMISSION DATE / POSTED DATE

20-08-2021 / 27-08-2021

\section{CITATION}

Du, Linsong; Ma, Jianhui; Fan, Chao; Liang, Qingpeng; Li, Chenxing; Tang, Youxi (2021): Spatial Domain Suppression of Co-Channel Interference: A Reflection Amplifiers Surface (RAS) Approach. TechRxiv. Preprint. https://doi.org/10.36227/techrxiv.15677823.v1

DOI 


\title{
Spatial Domain Suppression of Co-Channel
}

\section{Interference: A Reflection Amplifiers Surface (RAS) Approach}

\author{
Linsong Du, Student Member, IEEE, Jianhui Ma, Member, IEEE, Chao Fan, \\ Qingpeng Liang Member, IEEE, Chenxing Li, Youxi Tang, Member, IEEE
}

\begin{abstract}
This paper adopts a novel reflection amplifiers surface (RAS) to suppress the co-channel interference in the spatial domain. The RAS can reflect and amplify the electromagnetic wave with phase shifts by designing the reflection coefficients, which enables it more flexibly reconfigure the wireless propagation environment, and even suppress interference channel gain. In this paper, a transmitter and an interferer send the desired signal and interference to the receiver, respectively, and a RAS is placed to suppress the unknown interference. First, we design the reflection coefficients for optimizing the interference suppression ratio, and prove that when the number of reflection amplifiers is greater than the number of antennas at the interferer, the interference can be perfectly suppressed. Next, a capacity maximization problem is formulated to design the optimal reflection coefficients, and an iterative algorithm based on fractional programming and the convex-concave procedure is proposed to obtain the solution for this problem. Moreover, the closed-form expression of the maximal capacity is obtained in the strong interference power case. In addition, this paper shows the upper and lower boundaries of the maximal capacity and discusses what kind of the channel conditions achieve the upper and lower boundaries. Lastly, the above results are generalized to the multiple interferer scenario.
\end{abstract}

\section{Index Terms}

This work was supported by the National Key R\&D Program of China under grant 2018YFB1801903, the National Natural Science Foundation of China under grants 61901396, 62071094, U19B2014, 61771107, 61701075, 61601064, 61531009, and Sichuan Science and Technology Program 2020YFH0101.

L. Du, J. Ma, C. Li, and Y. Tang are with the National Key Laboratory of Science and Technology on Communications, University of Electronic Science and Technology of China, Chengdu, China, 611731. Emails: linsongdu@outlookcom, jianhuima@std.uestc.edu.cn, chenxingcd@gmail.com, tangyx@uestc.edu.cn.

Chao Fan is with CORPRO technology Co. Ltd., Chengdu, China 610041. Email: fanchao41@163.com.

Qingpeng Liang is with the School of Information Science and Technology, Southwest Jiaotong University, Chengdu, 611756, China. Email: qingpengliang@gmail.com. 
Reflection amplifier surfaces (RAS), Spatial Domain, Interference Suppression, Capacity Characterization.

\section{INTRODUCTION}

In the practical wireless communication scenarios, there is an almost inevitable issue that the receivers suffer co-channel interference from the interferers [1]-[7]. For instance, in the 5G ultradense cellular networks, considering the shortage of spectrum resources, it is deemed inevitable that the different base stations (BSs) work at the same time-frequency range, i.e., frequency reuse. Besides, the ultra-dense and small cell concept implies that the cell edge mobile users are close to the adjacent cell BSs [1]. Thus, they will suffer the co-channel interference from the adjacent cell BSs [2]. In addition, full-duplex technology brings the self-interference, which is also a pretty typical co-channel interference [3]. Moreover, except for the civilian areas, in confrontational battlefield environments, the military communication devices not only have a high probability of receiving the interference from hostile jamming interferers, but also may be jammed by the non-malicious non-stationary command post-co-site interference [4]-[7]. The cochannel interference will significantly reduce the signal to interference plus noise ratio (SINR), and thus raises the bit error rate or drops the achievable rate. As a result, it is hard to satisfy the demand of the higher data rate and high quality in wireless communications.

\section{A. Conventional Interference Suppression Technique}

Considering the mentioned issues in realistic communication scenarios, the co-channel interference suppression techniques, i.e., interference cancellation (IC) and interference alignment (IA), have been received widespread concern [8]-[15]. The conventional IC technique is to regenerate the interference signal and then subtract it from the received signals in the radio frequency (RF) and digital domains. However, it implies that the receiver must know the reference interference in advance, which is impractical in many wireless communication scenarios [8]-[10]. Furthermore, the receiver needs to estimate and reconstruct the interference channel by taps [9], [10]. Unfortunately, for the multiple antenna systems, the complexity of the interference channel reconstruction (i.e., the number of taps for channel reconstruction) in RF domain geometrically grows with the number of the antennas [10].

Therefore, the IA technique, which is a spatial domain interference suppression method, is proposed in multiple antennas communication systems [11]-[15]. The interferers design the 
precoding matrixes to regulate the interference vector falling into the interference subspace at the receiver while ensuring the desired signal vector is independent of interference subspace. Then, the receiver designs its filter matrix to zero-force the interference without desired signal. Comparing with IC, the IA technique allows receivers to be unknown about the interference signal. On the other hand, since the IA technology requires each interferer to design the precoding matrix, these interferers should cooperate with the receivers, which is difficult to be realized in many scenarios, especially for the cases with non-cooperative or hostile interferers [16]. Even though the interferers are partners, there is a trade-off between aligning interference and enhancing the desired signal for the interferers since interferers are also transmitters in some cases [17]. Moreover, under some channel conditions [17], the interferers or the receivers cannot find the appropriate precoding or unitary filter matrixes to zero-force the interference.

\section{B. Interference Suppression by Reconfigurable Intelligent Surface (RIS)}

To overcome the shortcomings of the conventional interference suppression methods, a novel device, i.e., reconfigurable intelligent surface, is proposed to suppress the co-channel interference [18]-[20]. The reconfigurable intelligent surface (RIS) consists of many passive reflectors, each of which can reflect the electromagnetic wave with the phase shift. Thus, the RIS can reflect the scattering interference with the phase shift to partly cancel the interference on the direct link in the spatial domain. In other words, the RIS is an approach to partly reconfiguring the channel of the interferer-RIS-receiver link, thereby partly controlling the interference propagation environment. Since the interference signal is suppressed by reducing the interference channel gain rather than designing the precoding matrixes at interferers, this way does not need the cooperation of the interferers and acquisition of the interference signal in advance. Moreover, the RIS does not need the complex RF chain, and thus it can be easily decorated in many places [21]. However, the RIS as a passive device cannot actively adjust the amplitude of the electromagnetic wave [21]. It indicates that the RIS cannot fully modify the channel of the interferer-RIS-receiver link, and the flexibility of controlling the interference propagation environment is limited. Therefore, the effectiveness of the interference suppression highly depends on the locations of the transmitters, interferers, receivers, and RIS. It is almost impossible that the RIS perfectly suppresses the interference in most communication scenarios. Inspire by the advantages and flaws of the RIS, this paper adopts a state-of-the-art active RIS, i.e., reflection amplifiers surface (RAS), to suppress the interference. 


\section{The Concept of Interference Suppression via RAS}

The RAS equips with multiple reflection amplifiers, where the reflection amplifiers can reflect and amplify the electromagnetic wave with the phase shift. The amplification capability of the reflection amplifier is mainly due to its negative resistance component, i.e., tunnel diode, which can convert direct current (DC) bias power into the RF power, thereby amplifying the received electromagnetic wave [22]. The reflection amplifier has been widely used in backscatter communications [22]-[24]. Similar to the passive reflectors at RIS, the amplifier reflectors do not have a complex RF chain, and thus they can be deployed on a surface in abundance. Therefore, the authors in [25] first proposed the RAS concept to assist wireless communications, where the RAS reflects and amplifies the scattering signal with phase shifts to increase the achievable rate. Comparing with RIS, the RAS offers more flexibility to adjust the wireless propagation environment. Therefore, the RAS is a perfect device to reconfigure the interference propagation environment.

The RAS-based interference suppression approach is to adjust the interferer-RAS-receiver channel vector that has the same norm as the interferer-receiver channel vector but in the opposite direction. It will lead the interference channel gain to become zero, thereby perfectly suppressing the interference. From the point of the interference signal, the RAS utilizes the modified scattering interference to counteract the interference on the direct path in the spatial domain to achieve "exploiting interference to suppress itself". The RAS-based approach is applicable to cases with the non-cooperative interferer, since this approach does not depend on the interferer. In other words, the interferer can arbitrarily design its signal (e.g., precoding matrix) without affecting the receiver.

Furthermore, the RAS not only can suppress the interference channel gain, but also can strengthen the transmission channel gain (i.e., the transmitter-RAS-receiver and transmitterreceiver links). Thus, it is also a fantastic way to improve the SINR and the capacity of the communication in the presence of interferer. Notice that a tradeoff may exist between the transmission channel gain enhancement and the interference channel gain suppression in some channel conditions. It brings more challenges in the reflection coefficients design on the RAS.

\section{Summary of Main Results}

In this paper, we propose a co-channel interference suppression approach based on RAS. In this system model, a transmitter with multiple antennas sends the signal to the receiver in the 
presence of the multiple antennas interferers. A RAS consisting of reflection amplifiers is installed near the receiver to suppress the interference and enhance the desired signal by designing its reflection coefficients. These channel models for the proposed wireless communication can be equivalent to a multiple-input single-output (MISO) channel in the interference system, where the transmission and interference links can both be controlled by the RAS. Thus, it is crucial to find the reflection coefficient designs for improving the performance of the equivalent MISO channel in the interference system.

The main contributions are summarized as follows:

- First, the reflection coefficients are designed to optimize the interference suppression ratio (ISR). The ISR minimization problem can be transformed into a least-squares problem, which is convex and thus can be solved. In addition, we prove that when the number of reflection amplifiers is greater than the number of antennas at the interferers, the interference can be perfectly suppressed. Even though the number of reflection amplifiers is less than the number of antennas at the interferers, the diversity order of the interferer-receiver link can be effectively counteracted by the RAS.

- Then, we formulate a channel capacity maximization problem for the RAS-assisted communication in the presence of the interferer to design the optimal reflection coefficients so that RAS can enhance the desired signal and suppress the interference, simultaneously. Since this problem is non-convex, we proposed an iterative algorithm based on the fractional programming and convex-concave procedure (CCP) method to solve it.

- Moreover, to acquire deeper insights on the capacity characterization, we obtain the closedform expression for the maximal capacity with interference in the strong interference power case. It concludes that optimizing the ISR is almost equivalent to maximize the capacity in the strong interference power case. Then, we show the upper and lower boundaries of the maximal capacity with interference, and then discuss what kind of the channel conditions can achieve them and show the corresponding optimal reflection coefficients design.

- Finally, this paper generalizes the above results to the multiple interferers scenario. We prove that if the number of reflection amplifiers is greater than the total numbers of the antennas at all interferers, the interferences can be perfectly suppressed. The proposed iterative algorithm is also be used for the multiple interferers scenario. We also present the upper and lower boundaries of the maximal capacity with interference for the multiple interferers scenario. 


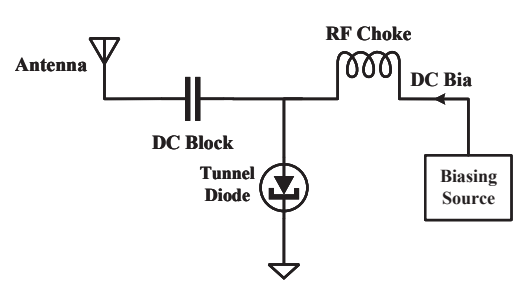

Fig. 1. Block diagram of a reflection amplifier.

The structure of this paper is organized as follows. Section II introduces the RAS in brief, and presents the signal model. Section III designs the reflection coefficients for minimizing the ISR. Section IV designs the reflection coefficients for maximizing the capacity, and analyzes the capacity characterization. Section V extends the results to multiple interferers scenario. Section VI numerical results are presented to show the performances. Section VII concludes our works.

Notation: $\mathbf{a}$ is a vector, $\mathbf{A}$ is a matrix. $\|\mathbf{a}\|$ is Euclidean norm of $\mathbf{a} . \mathbf{A}^{H}$ and $\mathbf{A}^{-1}$ denote Hermitian transpose and pseudo-inverse of $\mathbf{A}$, respectively. $\operatorname{diag}(\mathbf{a})$ is a diagonal matrix with the entries of a on its main diagonal. $\mathbb{C}^{N \times M}$ denotes the set of all $N \times M$ complex-valued matrices. $\mathbf{I}_{N}$ represents $N \times N$ identity matrix. $\mathbf{0}_{M \times N}$ represents $M \times N$ null matrix. $j \triangleq \sqrt{-1}$ is the imaginary unit.

\section{System MODEL}

This paper proposes a RAS-based co-channel interference spatial domain suppression approach in wireless communication. Before we introduce the signal model of the proposed communication system, we first show how the revolutionary device, i.e., RAS, works in brief.

\section{A. Preliminary}

The RAS consists of multiple reflection amplifiers, each of which can reflect and amplify the electromagnetic wave with the phase shift. The block diagram of a reflection amplifier is shown in Fig. 1 [22]. The reflection coefficient of the reflection amplifier is determined by the impedance of its antenna and load:

$$
\alpha=\frac{Z_{L}-Z_{A}}{Z_{L}+Z_{A}}
$$




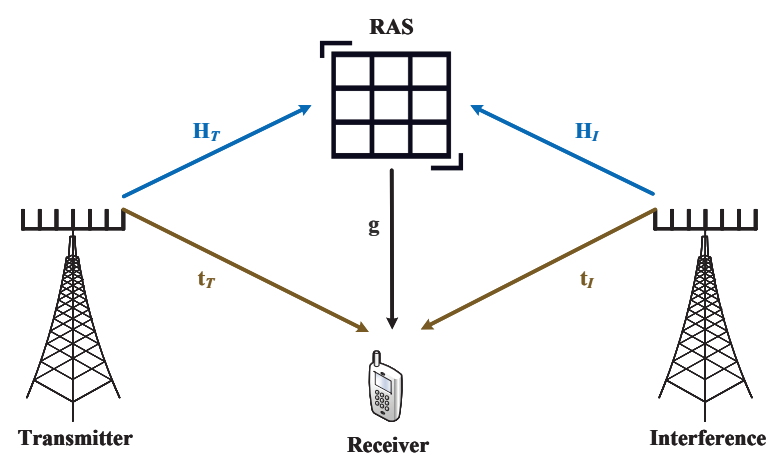

Fig. 2. RAS-assisted communication in presence of an interferer.

where $Z_{L}$ and $Z_{A}$ are impedances of the load and the antenna, respectively. First, the impedance of the antenna is characterized by

$$
Z_{A}=R_{A}+j X_{A}
$$

where the real part $R_{A}>0$ and the imaginary part $j X_{A}$ represent the resistance and the reactance of the antenna, respectively. Then, at the cost of a certain amount of biasing voltage, the impedance of the load is characterized by

$$
Z_{L}=-R_{L}+j X_{L}
$$

where the real part $-R_{L}<0$ and the imaginary part $j X_{L}$ represent the negative resistance and the reactance of the load, respectively. It is worth noticing that the load shows the negative impedance characteristic, due to negative resistance effect of the tunnel diode in the load. Thus, it can be observed that the reflection gain is greater than 1:

$$
|\alpha|^{2}=\frac{\left(R_{L}+R_{A}\right)^{2}+\left(X_{L}+X_{A}\right)^{2}}{\left(R_{L}-R_{A}\right)^{2}+\left(X_{L}+X_{A}\right)^{2}}>1 .
$$

By modulating the load impedance, the reflection amplifier can control the reflection coefficient. In fact, the amplification capability is due to the fact that the load converts the DC biasing power into the RF power. Moreover, since the reflection amplifier cannot support the high reflection gain for the high input power of the signal, the RAS should be placed near the receiver and far away from the interferer and the transmitter. 


\section{B. Signal Model}

As illustrated in Fig. 2, a transmitter with multiple-antennas transmits the desired signal vector $\mathbf{x}_{T} \in \mathbf{C}^{M_{T} \times 1}$ to the receiver with single antenna, where $M_{T}$ is the number of antennas at the transmitter. At the same time, an interferer sends the interference vector $\mathbf{x}_{T} \in \mathbf{C}^{M_{I} \times 1}$ to the receiver, where $M_{T}$ is the number of antennas at the interferer. In order to suppress the interference, a RAS with $N$ reflection amplifiers is installed near the receiver. The received signal at the receiver is given as

$$
\begin{aligned}
y & =\mathbf{t}_{T}^{H} \mathbf{x}_{T}+\mathbf{t}_{I} \mathbf{x}_{I}+\mathbf{g}^{H} \mathbf{\Phi}\left(\mathbf{H}_{T} \mathbf{x}_{T}+\mathbf{H}_{I} \mathbf{x}_{I}+\mathbf{z}_{1}\right)+z_{0} \\
& =\underbrace{\left(\mathbf{t}_{T}^{H}+\mathbf{g}^{H} \boldsymbol{\Phi} \mathbf{H}_{T}\right) \mathbf{x}_{T}}_{\text {Desired Signal }}+\underbrace{\left(\mathbf{t}_{I}^{H}+\mathbf{g}^{H} \boldsymbol{\Phi} \mathbf{H}_{I}\right) \mathbf{x}_{I}}_{\text {Interference }}+\underbrace{\mathbf{g}^{H} \mathbf{\Phi} \mathbf{z}_{1}+z_{0}}_{\text {Noise }},
\end{aligned}
$$

where $\mathbf{t}_{T}^{H} \in \mathbb{C}^{1 \times M_{T}}$ is the channel vector between the transmitter and the receiver; $\mathbf{t}_{I}^{H} \in \mathbb{C}^{1 \times M_{I}}$ is the channel vector between the interferer and the receiver; $\mathbf{H}_{T} \in \mathbb{C}^{N \times M_{T}}$ is the channel matrix between the transmitter and the RAS; $\mathbf{H}_{I} \in \mathbb{C}^{N \times M_{I}}$ is the channel matrix between the interferer and the RAS; $\mathbf{g}^{H} \in \mathbb{C}^{1 \times N}$ is the channel vector between the RAS and the receiver; $\boldsymbol{\Phi}=\operatorname{diag}\left[\alpha_{1}, \alpha_{2}, \cdots, \alpha_{N}\right] \in \mathbb{C}^{N \times N}$ is a diagonal matrix denoting the reflection coefficients of the reflection amplifiers at the RAS, $\alpha_{n}$ indicates the phase shift and the amplitude amplifying for the received signals at $n$-th reflection amplifier; $z_{0}$ is the circularly symmetric complex Gaussian (CSCG) noise with zero mean and variance $\sigma^{2}$ at the receiver; and $\mathbf{z}_{1}$ is the CSCG vector with $\mathcal{C N}\left(\mathbf{0}, \sigma^{2} \mathbf{I}\right)$ in the RAS.

$\mathbf{C}_{T}=\mathbf{G H}_{T}$ denote the cascaded channel from the transmitter to the receiver via RAS and $\mathrm{C}_{I}=\mathbf{G H}_{I}$ to represent the cascaded channel from the interferer to the receiver via RAS, where $\mathbf{G}=\operatorname{diag}(\mathbf{g})$. We define $\mathbf{a}=\left[\alpha_{1}, \alpha_{2}, \cdots, \alpha_{N}\right]^{T}$. Then, signal model (6) is equivalent to

$$
y=\underbrace{\left(\mathbf{t}_{T}^{H}+\mathbf{a}^{H} \mathbf{C}_{T}\right) \mathbf{x}_{T}}_{\text {Desired Signal }}+\underbrace{\left(\mathbf{t}_{I}^{H}+\mathbf{a}^{H} \mathbf{C}_{I}\right) \mathbf{x}_{I}}_{\text {Interference }}+\underbrace{\mathbf{a}^{H} \mathbf{G z}_{1}+z_{0}}_{\text {Noise }} .
$$

Remark 2.1: Based on (7), we can observe that the channel coefficients of the transmission and interference links and can both be controlled by the reflection coefficient a. Thus, the interference signal can be suppressed by zero-forcing interference channel gain. Notice that this spatial domain interference suppression scheme does not know the interference signal $\mathbf{x}_{I}$.

Remark 2.2: It is different from the conventional IA that the RAS is to suppress the interference channel gain without the signal design at the interferer (e.g., the precoding matrixes design at 
the interferer). Thus, without loss of generality, the worst case of covariance matrix of the interference vector $\mathbf{x}_{I}$ given as $\mathbf{Q}_{I}^{*}=P_{I} \frac{\left(\mathbf{t}_{I}+\mathbf{H}_{I}^{H} \mathbf{\Phi}^{H} \mathbf{g}\right)\left(\mathbf{g}^{H} \mathbf{\Phi} \mathbf{H}_{I}+\mathbf{t}_{I}^{H}\right)}{\left\|\mathbf{g}^{H} \mathbf{\Phi} \mathbf{H}_{I}+\mathbf{t}_{I}^{H}\right\|^{2}}$ is considered in the rest of this paper [26], [27], where $P_{I}=\mathbb{E}\left\{\mathbf{x}_{I}^{H} \mathbf{x}_{I}\right\}$ is the interference power. Under this covariance matrix, the power of the received interference is maximal.

\section{REFLECTION COEFFICIENTS DESIGN FOR ISR}

RAS can effectively suppress the interference by controlling the channel conditions of interference links via reflection coefficients. Thus, in this section, the reflection coefficients design is considered for suppressing the interference. We firstly formulate an ISR minimization problem, which is convex. Then, the optimal reflection coefficients design is obtained for this problem. For some cases, the optimal reflection coefficients design is closed-form.

\section{A. ISR Minimization Problem}

First, the maximal power of the received interference without RAS is given as $P_{I}\left\|\mathbf{t}_{I}^{H}\right\|^{2}$, and when the RAS is installed, the maximal power of the received interference is rewritten as $P_{I}\left\|\mathbf{t}_{I}^{H}+\mathbf{a}^{H} \mathbf{C}_{I}\right\|^{2}$ [28]. Thus, we can obtain the ISR:

$$
G(\mathbf{a})=\frac{P_{I}\left\|\mathbf{t}_{I}^{H}+\mathbf{a}^{H} \mathbf{C}_{I}\right\|^{2}}{P_{I}\left\|\mathbf{t}_{I}^{H}\right\|^{2}} .
$$

Obviously. the lower ISR implies the better interference suppression performance. Thus, an ISR minimization problem is formulated as

$$
\begin{array}{ll}
(\mathrm{P} 1) & \min _{\mathbf{a}} G(\mathbf{a}) \\
& \text { s.t. }\left|\alpha_{n}\right| \leq \alpha_{\max }, n=1, \cdots, N,
\end{array}
$$

where $\alpha_{\max }>0$ is the maximum amplitude that the reflection amplifier can provid.

\section{B. Reflection Coefficients Design for Problem (P1)}

Problem (P1) is quadratic optimization problem, and thus optimally numerical solution can be solved by the CVX tool. In order to obtain the optimal closed-form solution for some special cases. We first investigate the unconstrained problem of Problem (P1):

$$
(\mathrm{P} 1 *) \min _{\mathbf{a}} G(\mathbf{a})
$$


which is a least-squares problem. The least-squares problem may has multiple optimal closedform solutions, i.e., least-squares solutions. We define solution set of Problem (P1) as $\Psi$. To satisfy constraint condition (10) as best as we can, we choose the minimal norm solution of least-squares solutions as the optimal reflection coefficients design for Problem $(\mathrm{P} 1 *)$, which is denoted by

$$
\hat{\mathbf{a}}=\arg \min _{\mathbf{a} \in \Psi}\{\|\mathbf{a}\|\} .
$$

The form of the minimal norm solution $\hat{\mathbf{a}}$ is given as [29]

$$
\hat{\mathbf{a}}=-\mathbf{D}^{H}\left(\mathbf{D D}^{H}\right)^{-1}\left(\mathbf{B}^{H} \mathbf{B}\right)^{-1} \mathbf{B}^{H} \mathbf{t}_{I}
$$

where $\mathbf{D} \in \mathbb{C}_{r}^{r \times N}$ and $\mathbf{B} \in \mathbb{C}_{r}^{M_{I} \times r}$ are obtained by the full rank decomposition for $\mathbf{C}_{I}^{H}$, i.e., $\mathbf{C}_{I}^{H}=\mathbf{B D}$, and $r$ is the rank of $\mathbf{C}_{I}^{H}$.

After obtaining the optimal reflection coefficients design â for Problem $(\mathrm{P} 1 *)$, we verify that $\hat{\mathbf{a}}$ whether satisfies constraint condition (10): 1) If â satisfies constraint condition (10), $\hat{\mathbf{a}}$ is optimal reflection coefficients design for Problem (P1);2) if â does not satisfy constraint condition (10), $\hat{\mathbf{a}}$ is not the optimal reflection coefficients design for Problem (P1), and the optimal reflection coefficients design for Problem (P1) is computed by CVX tool. The details are summarized in Algorithm 1.

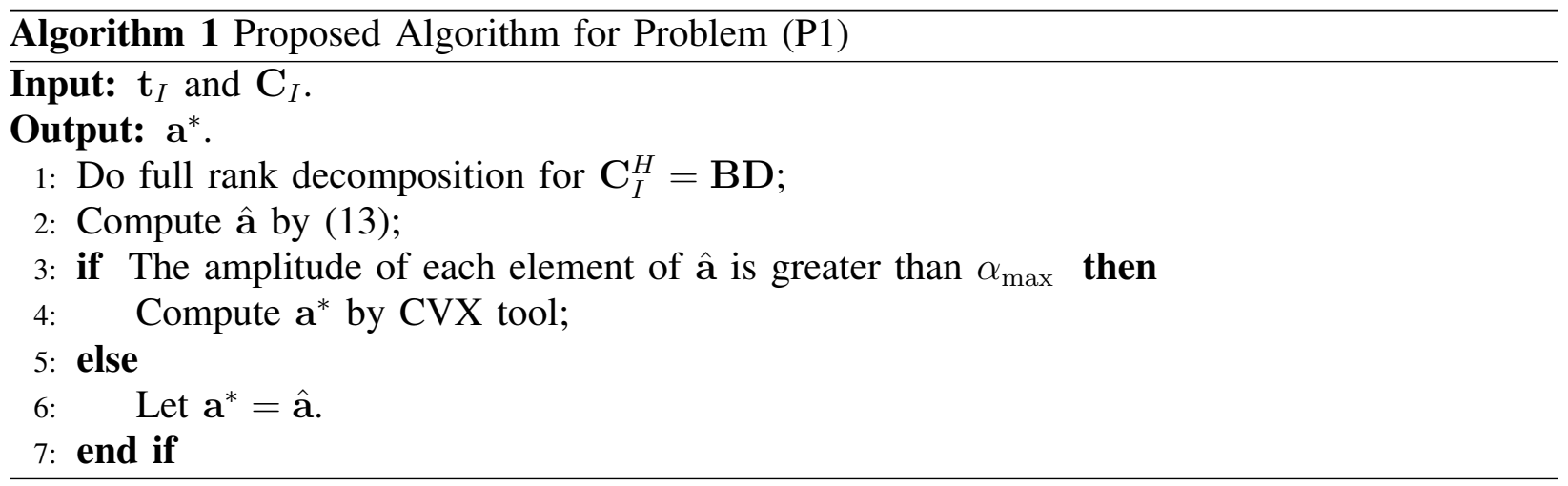

Remark 3.1: Here, we discuss the complexity of Algorithm 1. First, the complexity of computing $\hat{\mathbf{a}}$ is mainly due to the calculations of full rank decomposition for $\mathbf{C}_{I}^{H}$ and (13), whose complexities are $\mathcal{O}\left(M_{I} N^{2}\right)$ and $\mathcal{O}\left(N r^{2}+M_{I} r^{2}+r M_{I} N\right)$. It follows that the complexity of computing $\hat{\mathbf{a}}$ is given as $\mathcal{O}\left(N r^{2}+M_{I} r^{2}+r M_{I} N+M_{I} N^{2}\right)$. If $\hat{\mathbf{a}}$ does not satisfy constraint condition (10), we compute $\mathbf{a}^{*}$ by the CVX tool [30], whose complexity is given as $\mathcal{O}\left(N^{2} S\right)$, 
where $S$ is the number of iterations.

\section{Minimal ISR of Special Cases}

In this subsection, we show the expressions of the minimal ISR for two special cases and discuss the diversity order.

Row Full Rank Case: If $\mathbf{C}_{I}^{H}$ is row full rank, i.e., $N \geq M_{I}=r$, and supposing constraint condition (10) is satisfied, the optimal reflection coefficients design for Problem (P1*) given in (13) can be rewritten as

$$
\hat{\mathbf{a}}=-\mathbf{C}_{I}\left(\mathbf{C}_{I}^{H} \mathbf{C}_{I}\right)^{-1} \mathbf{t}_{I}
$$

For this case, the optimal value for Problem $\left(\mathrm{P} 1^{*}\right)$ is $\min _{\mathbf{a}} G(\mathbf{a})=0$, which implies that the interference can be completely suppressed.

Column Full Rank Case: If $\mathbf{C}_{I}^{H}$ is column full rank, i.e., $M_{I} \geq N=r$, and supposing constraint condition (10) is satisfied, the optimal reflection coefficients design for Problem (P1*) given in (13) can be rewritten as

$$
\hat{\mathbf{a}}=-\left(\mathbf{C}_{I} \mathbf{C}_{I}^{H}\right)^{-1} \mathbf{C}_{I} \mathbf{t}_{I}
$$

Moreover, we discuss the optimal value of Problem (P1*) under the column full rank case. First, we define $\overline{\mathbf{t}}_{I}=\mathbf{V}_{I}^{H} \mathbf{t}_{I}=\left[\bar{t}_{1}, \bar{t}_{2}, \cdots, \bar{t}_{M_{I}}\right]$, where $\mathbf{V}_{I}^{H} \in \mathbb{C}^{M_{I} \times M_{I}}$ is an unitary matrix obtained by the singular value decomposition (SVD) of $\mathbf{C}_{I}$, i.e., $\mathbf{C}_{I}=\mathbf{U}_{I} \Sigma_{I} \mathbf{V}_{I}^{H}$ and $\Sigma_{I} \in \mathbb{C}^{N \times M_{I}}$ is a rectangular matrix whose diagonal elements are non-negative real numbers and whose other elements are zero. Then, the optimal value under column full rank case is given as the following proposition.

Proposition 3.1: The optimal value of Problem $\left(\mathrm{P} 1^{*}\right)$ under the column full rank case is given as

$$
\min _{\mathbf{a}} G(\mathbf{a})=\frac{\sum_{m=N+1}^{M_{I}}\left|\bar{t}_{m}\right|^{2}}{\left\|\overline{\mathbf{t}}_{I}^{H}\right\|^{2}}
$$

Proof: See Appendix A.

Remark 3.2: From Proposition 3.1, we obtain the minimal ISR under column full rank case. It can be observed that the minimal amount of diversity for interference links $\left(\mathbf{t}_{I}^{H}+\mathbf{a}^{H} \mathbf{C}_{I}\right)$ is $\left(M_{I}-N\right)^{+}$. Since without RAS, the amount of diversity for $\mathbf{t}_{I}^{H}$ is $M_{I}$, the decreased amount of 
diversity for $\mathbf{t}_{I}$ is equal to the number of reflection amplifiers $N$. When the number of reflection amplifiers is greater than the amount of diversity for $\mathbf{t}_{I}$, the amount of diversity is zero, i.e., row full rank case.

\section{REFLECTION COEFFICIENTS DESIGN FOR CAPACITY WITH INTERFERENCE}

The RAS not only can suppress the interference links but also reconfigure the transmission links. Thus, only focusing on suppressing the interference may decrease the desired signal. Considering this issue, this section designs the reflection coefficients for the capacity of the RAS assisted communication with an interferer. We first formulate a capacity maximization problem. Then, since the proposed problem is non-convex, we adopt fractional programming and the CCP method to solve it.

\section{A. Capacity Maximization Problem}

Based on the signal model (7), for the fixed reflection coefficients a, the capacity of the RAS-assisted communication system with interference is given as [26], [27]

$$
C(\mathbf{a})=\log \left(1+\frac{P_{T}\left\|\mathbf{t}_{T}^{H}+\mathbf{a}^{H} \mathbf{C}_{T}\right\|^{2}}{P_{I}\left\|\mathbf{t}_{I}^{H}+\mathbf{a}^{H} \mathbf{C}_{I}\right\|^{2}+\sigma^{2}\left\|\mathbf{a}^{H} \mathbf{G}\right\|^{2}+\sigma^{2}}\right),
$$

where $P_{T}=\mathbb{E}\left\{\mathbf{x}_{T}^{H} \mathbf{x}_{T}\right\}$ is the transmission power at the transmitter. Obviously, the capacity of the RAS assisted communication with an interferer depends on the reflection coefficients $\mathbf{a}$. To obtain the optimal reflection coefficients $\mathbf{a}^{*}$ for both interference suppression and desired signal enhancement, a capacity maximization problem is formulated as

$$
\begin{gathered}
\max _{\mathbf{a}} C(\mathbf{a}) \\
\text { s.t. }(10) .
\end{gathered}
$$

Since the function $\log (\cdot)$ is monotonically increasing, the capacity maximization is equivalent to SINR maximization, i.e.,

$$
\text { (P2) } \gamma^{*}=\max _{\mathbf{a}} \frac{P_{T}\left\|\mathbf{t}_{T}^{H}+\mathbf{a}^{H} \mathbf{C}_{T}\right\|^{2}}{P_{I}\left\|\mathbf{t}_{I}^{H}+\mathbf{a}^{H} \mathbf{C}_{I}\right\|^{2}+\sigma^{2}\left\|\mathbf{a}^{H} \mathbf{G}\right\|^{2}+\sigma^{2}}
$$

s.t. $(10)$, 
where $\gamma^{*}$ is the optimal value for Problem (P2). It is obvious that constrain (10) for Problem (P2) is convex; however, the objective function (19) is non-convex. Thus, Problem (P2) is nonconvex. The optimal reflection coefficients $\mathbf{a}^{*}$ cannot be directly computed by a standard and effective method.

\section{B. Reflection Coefficients Design for Problem (P2)}

Since Problem (P2) is non-convex, we solve Problem (P2) by fractional programming and CCP method [31].

First, we formulate Problem (P2*):

$$
\begin{aligned}
\left(\mathbf{P} 2^{*}\right) f(\gamma)=\min _{\mathbf{a}} \gamma\left(P_{I}\left\|\mathbf{t}_{I}^{H}+\mathbf{a}^{H} \mathbf{C}_{I}\right\|^{2}+\sigma^{2}\left\|\mathbf{a}^{H} \mathbf{G}\right\|^{2}+\sigma^{2}\right)-P_{T}\left\|\mathbf{t}_{T}^{H}+\mathbf{a}^{H} \mathbf{C}_{T}\right\|^{2} \\
\text { s.t. }(10),
\end{aligned}
$$

and define $\mathbf{a}^{(\gamma)}$ as the solution for Problem (P2*) with fixed $\gamma$.

Lemma 4.1: The maximal value $\gamma^{*}$ is the root of equation $f(\gamma)=0$. When $\gamma=\gamma^{*}$, Problems (P2) and (P2*) own same optimal solution, i.e., $\mathbf{a}^{*}=\mathbf{a}^{\left(\gamma^{*}\right)}$.

Proof: Please see [32].

Remark 4.1: It is observed that $f(\gamma)$ is monotonically increasing with $\gamma$. Therefore, we can obtain $f(\gamma)=0$ by bisection search over $\gamma$. In each iteration, we solve Problem (P2*) for the fixed $\gamma$. Accordingly, we will focus on Problem $\left(\mathrm{P} 2^{*}\right)$ in the sequel.

1) Solving Problem (P2*) by CCP method: However, Problem (P2*) is still non-convex, since the first item of the objective function in (20) is convex and the second item of the objective function in (20) is concave. Thus, it cannot be directly solved either by a standard method. Considering the convex-concave structure of the objective function in (20), we adopt the CCP method to dispose it.

Next, we show how to solve Problem $(\mathrm{P} 2 *)$ by the CCP method. The CCP method is an iterative approach. In each iteration, we solve an approximation of Problem (P2*). The optimal solution of the approximation problem is considered as a parameter for the approximation problem in the next iteration. In the $i$-th iteration, by linearizing the second item of (20), i.e., $-P_{T}\left\|\mathbf{t}_{T}^{H}+\mathbf{a}^{H} \mathbf{C}_{T}\right\|^{2}$, the objective function in (20) for Problem (P2*) can be linearly approximated as

$g\left(\mathbf{a}, \mathbf{a}_{i-1}\right)=\gamma\left(P_{I}\left\|\mathbf{t}_{I}^{H}+\mathbf{a}^{H} \mathbf{C}_{I}\right\|^{2}+\sigma^{2}\left\|\mathbf{a}^{H} \mathbf{G}\right\|^{2}+\sigma^{2}\right)-2 P_{T}\left(\mathbf{a}^{H}-\mathbf{a}_{i-1}^{H}\right)\left(\mathbf{C}_{T} \mathbf{C}_{T}^{H} \mathbf{a}_{i-1}+\mathbf{C}_{T} \mathbf{t}_{T}\right)$ 


$$
+P_{T}\left\|\mathbf{t}_{T}^{H}+\mathbf{a}_{i-1}^{H} \mathbf{C}_{T}\right\|^{2}
$$

where $\mathbf{a}_{i-1}$ is the optimal solution at the $(i-1)$-th iteration, i.e., the previous iteration. Obviously, for the fixed $\mathbf{a}_{i-1}$, the function $g\left(\mathbf{a}, \mathbf{a}_{i-1}\right)$ is convex over $\mathbf{a}$. Based on (21), we obtain the following problem

$$
\left(\mathrm{P} 2 *_{-i}\right) \quad \mathbf{a}_{i}=\arg \min _{\mathbf{a}} g\left(\mathbf{a}, \mathbf{a}_{i-1}\right)
$$

s.t. $(10)$,

where $\mathbf{a}_{i}$ is the optimal solution at the $i$-th iteration, and it can be computed by CVX tool [30]. Finally, $\mathbf{a}_{i}$ will converge a local optimal solution for Problem (P2) over $i$ [31].

2) Algorithm Summary: Based on above discussions, a two-level iterative algorithm is summarized in Algorithm 2 to search a feasible solution to Problem (P2). For inter-level, we adopt CCP method to solve Problem (P2*) for the fixed $\gamma$. In each iteration of CCP method, we compute $\mathbf{a}_{i}$ by CVX [30] and $\mathbf{a}_{i-1}$ which is obtained at the previous iteration. For the outer-level, we rely $\mathbf{a}^{(\gamma)}$ obtained in the inner iteration to compute $f(\gamma)$. Since $f(\gamma)$ is a monotonically increasing function of $\gamma, \gamma^{*}$ and $\mathbf{a}^{*}$ can also be obtained by the bisection research.

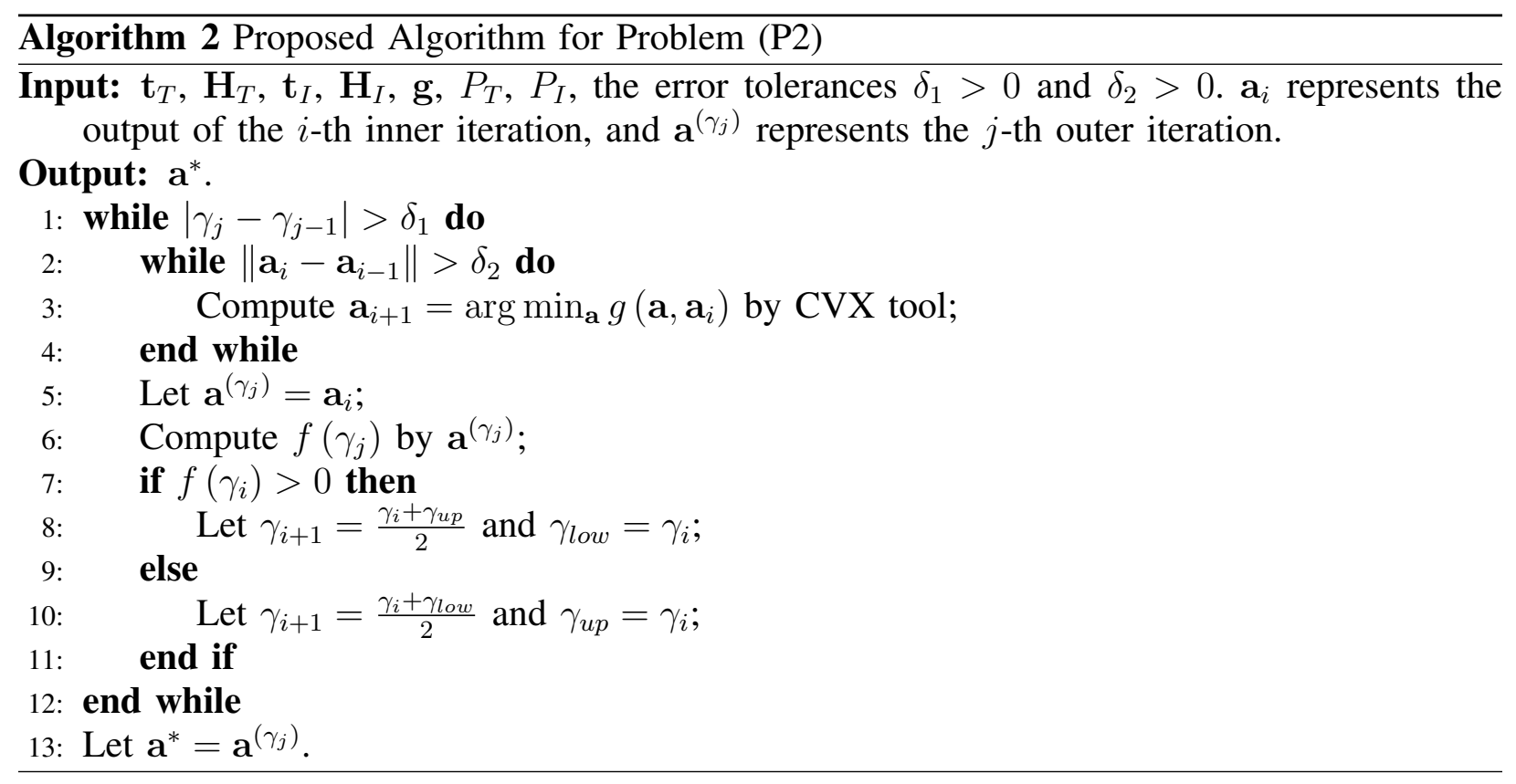

Remark 4.2: The complexity of Algorithm 2 is mainly due to the calculations of $\mathbf{a}_{i}$, whose complexity is $\mathcal{O}\left(\left(N^{2}+N\right) S\right)$ obtained by [30], where $S$ is the number of iterations for CVX. Then, the numbers of the inner and outer iterations are $I$ and $J$, respectively, and thus the 
complexity of Algorithm 2 is given as $\mathcal{O}\left(\left(N^{2}+N\right) S I J\right)$. In addition, the CCP and bisection method are both rapidly convergent [31], and thus the convergence rate of the Algorithm 2 is at least linear form.

\section{Capacity Characterization Analysis}

In the above subsection, we propose Algorithm 2 to optimize the capacity with interference. However, it loses some intuitions for the performances of the RAS assisted wireless communication with the interferer since we cannot obtain the closed-form expression of the maximal capacity with interference. Thus, this subsection firstly analyzes the capacity characterization in the strong interference power case, and then discusses the upper and lower boundaries of the maximal capacity with interference.

1) Strong Interference Power Case: Firstly, we consider a special case, i.e., strong interference power case, by letting $P_{I} \rightarrow \infty$.

Proposition 4.1: When $\mathbf{C}_{I}$ is a full rank matrix and $P_{I} \rightarrow \infty$, the optimal reflection coefficients for ISR minimization problem, i.e., $\mathbf{a}^{*}=-\left(\mathbf{C}_{I}^{H}\right)^{-1} \mathbf{t}_{I}$, is the same with that for capacity maximization problem. The corresponding maximal capacity with interference is given as

$$
\max C(\mathbf{a})=\log \left(1+\frac{P_{T}\left\|\mathbf{t}_{T}^{H}-\mathbf{t}_{I}^{H} \mathbf{C}_{I}^{-1} \mathbf{C}_{T}\right\|^{2}}{\sigma^{2}\left\|\mathbf{t}_{I}^{H} \mathbf{C}_{I}^{-1} \mathbf{G}\right\|^{2}+\sigma^{2}}\right)
$$

Proof: Since $\mathbf{C}_{I}$ is a full rank matrix, equation $\mathbf{C}_{I}^{H} \mathbf{a}+\mathbf{t}_{I}=\mathbf{0}$ owns the unique solution $\mathbf{a}^{*}=-\left(\mathbf{C}_{I}^{H}\right)^{-1} \mathbf{t}_{I}$, which is the optimal solution for Problem (P1). Thus, If $\mathbf{a} \neq-\left(\mathbf{C}_{I}^{H}\right)^{-1} \mathbf{t}_{I}$, it leads $\left\|\mathbf{t}_{I}^{H}+\mathbf{a}^{H} \mathbf{C}_{I}\right\|^{2}>0$, thereby $P_{I}\left\|\mathbf{t}_{I}^{H}+\mathbf{a}^{H} \mathbf{C}_{I}\right\|^{2} \rightarrow \infty$. It follows $C(\mathbf{a})=0$. Conversely, If $\mathbf{a}=-\left(\mathbf{C}_{I}^{H}\right)^{-1} \mathbf{t}_{I}$, we can obtain $C(\mathbf{a})=\log \left(1+\frac{P_{T}\left\|\mathbf{t}_{T}^{H}-\mathbf{t}_{I}^{H} \mathbf{C}_{I}^{-1} \mathbf{C}_{T}\right\|^{2}}{\sigma^{2}\left\|\mathbf{t}_{I}^{H} \mathbf{C}_{I}^{-1} \mathbf{G}\right\|^{2}+\sigma^{2}}\right)>0$. Thus, $\mathbf{a}^{*}=$ $-\left(\mathbf{C}_{I}^{H}\right)^{-1} \mathbf{t}_{I}$ is the optimal solution for Problem (P2).

Remark 4.3: Proposition 4.1 indicates that under the strong interference power case (e.g., selfinterference), optimizing the ISR is almost equivalent to maximize the capacity with interference. In other words, the RAS only needs to focus on interference suppression in the strong interference power case.

2) Upper Boundary: First, we show the upper boundary of the maximal capacity under the high SNR regime (e.g., the power of the desired signal and interference is high, or the distance between the transmitter and receiver is short). 
Lemma 4.2: When $\sigma^{2}\left\|\mathbf{g}^{H}\right\|^{2} \ll P_{T} \lambda_{T, \max }^{2}$, the upper boundary of the maximal capacity with interference is given as

$$
\max C(\mathbf{a}) \leq \log \left(1+\frac{P_{T}\left(\lambda_{T, \max }^{2} \alpha_{\max }^{2} N+2 \lambda_{T, \max } \alpha_{\max } \sqrt{N}\left\|\mathbf{t}_{T}^{H}\right\|+\left\|\mathbf{t}_{T}^{H}\right\|^{2}\right)}{N \alpha_{\max }^{2} \sigma^{2}\left\|\mathbf{g}^{H}\right\|^{2}+\sigma^{2}}\right)
$$

where $\lambda_{T, \max }$ is the maximal singular value of $\mathbf{C}_{T}$.

Proof: It is obvious that

$$
\begin{aligned}
& \frac{P_{T}\left\|\mathbf{t}_{T}^{H}+\mathbf{a}^{H} \mathbf{C}_{T}\right\|^{2}}{P_{I}\left\|\mathbf{t}_{I}^{H}+\mathbf{a}^{H} \mathbf{C}_{I}\right\|^{2}+\sigma^{2}\left\|\mathbf{a}^{H} \mathbf{G}\right\|^{2}+\sigma^{2}} \\
\leq & \frac{P_{T}\left(\mathbf{a}^{H} \mathbf{C}_{T} \mathbf{C}_{T}^{H} \mathbf{a}+2 \mathbf{t}_{T}^{H} \mathbf{C}_{T}^{H} \mathbf{a}+\left\|\mathbf{t}_{T}^{H}\right\|^{2}\right)}{\sigma^{2}\left\|\mathbf{a}^{H} \mathbf{G}\right\|^{2}+\sigma^{2}} \\
\leq & \frac{P_{T}\left(\lambda_{T, \max }^{2}\left\|\mathbf{a}^{H}\right\|^{2}+2 \lambda_{T, \max }\left\|\mathbf{t}_{T}^{H}\right\|^{2}\left\|\mathbf{a}^{H}\right\|+\left\|\mathbf{t}_{T}^{H}\right\|^{2}\right)}{\sigma^{2}\left\|\mathbf{a}^{H} \mathbf{G}\right\|^{2}+\sigma^{2}} \\
\leq & \frac{P_{T}\left(\lambda_{T, \max }^{2} \alpha_{\max }^{2} N+2 \lambda_{T, \max } \alpha_{\max } \sqrt{N}\left\|\mathbf{t}_{T}^{H}\right\|+\left\|\mathbf{t}_{T}^{H}\right\|^{2}\right)}{N \alpha_{\max }^{2} \sigma^{2}\left\|\mathbf{g}^{H}\right\|^{2}+\sigma^{2}}
\end{aligned}
$$

where

- (24) is due to $\mathbf{a}^{H} \mathbf{C}_{T} \mathbf{C}_{T}^{H} \mathbf{a} \leq \lambda_{T, \max }^{2}\left\|\mathbf{a}^{H}\right\|^{2}$ and $\mathbf{t}_{T}^{H} \mathbf{C}_{T}^{H} \mathbf{a} \leq \lambda_{T, \max }\left\|\mathbf{t}_{T}^{H}\right\|^{2}\left\|\mathbf{a}^{H}\right\|$ [29];

- (25) is due to $\left\|\mathbf{a}^{H}\right\|^{2} \leq \alpha_{\max }^{2} N$ from (10) and $\sigma^{2}\left\|\mathbf{g}^{H}\right\|^{2} \ll P_{T} \lambda_{T, \max }^{2}$.

Based on (25), (23) is obtained.

Then, we discuss what kind of channel conditions can achieve the upper boundary and the corresponding optimal reflection coefficients design. By the SVD, we obtain $\mathbf{C}_{T}=\mathbf{U}_{T} \Sigma_{T} \mathbf{V}_{T}^{H}$. Without loss of generality, the singular values of $\mathbf{C}_{T}$ are given as $\lambda_{T, 1} \geq \lambda_{T, 2} \geq, \cdots, \geq \lambda_{T, r}$ and please notice that $\lambda_{T, \max }=\lambda_{T, 1}$. At the same time, the singular values of $\mathbf{C}_{I}$ are given as $\lambda_{I, 1} \geq \lambda_{I, 2} \geq, \cdots, \geq \lambda_{I, r}$. Based on the above definitions, we can obtain the following proposition.

Proposition 4.2: Under the channel conditions of $\mathbf{t}_{T}^{H}=\left[\left\|\mathbf{t}_{T}\right\|, 0,0, \cdots\right] \mathbf{V}_{T}^{H}, \mathbf{U}_{T}=-\mathbf{U}_{I}$, and $\mathbf{t}_{I}^{H}=\left[\lambda_{I, 1} \alpha_{\max } \sqrt{N}, 0,0, \cdots\right] \mathbf{V}_{I}^{H}$, the maximal capacity with interference is given as

$$
\max C(\mathbf{a})=\log \left(1+\frac{P_{T}\left(\lambda_{T, \max }^{2} \alpha_{\max }^{2} N+2 \lambda_{T, \max } \alpha_{\max } \sqrt{N}\left\|\mathbf{t}_{T}^{H}\right\|+\left\|\mathbf{t}_{T}^{H}\right\|^{2}\right)}{N \alpha_{\max }^{2} \sigma^{2}\left\|\mathbf{g}^{H}\right\|^{2}+\sigma^{2}}\right),
$$


and the corresponding optimal reflection coefficients design is given as

$$
\mathbf{a}^{*}=\mathbf{U}_{T}\left[\sqrt{N} \alpha_{\max }, 0,0, \cdots\right]^{H} .
$$

Proof: See Appendix B.

Remark 4.4: From Proposition 4.2, we conclude that

- Under the above special channel condition, in order to achieve the upper boundary of the maximal capacity, the RAS must adjust the direction of the transmitter-RAS-receiver channel vector $\mathbf{C}_{T}^{H} \mathbf{a}^{*}$ to be the same with the direction of the transmitter-receiver channel vector $\mathbf{t}_{T}$, and the proof is given as

$$
\begin{aligned}
\mathbf{C}_{T}^{H} \mathbf{a}^{*} & =\mathbf{V}_{T} \Sigma_{T}^{H}\left[\sqrt{N} \alpha_{\max }, 0,0, \cdots\right]^{H} \\
& =\mathbf{V}_{T}\left[\lambda_{T, \max } \sqrt{N} \alpha_{\max }, 0,0, \cdots\right]^{H} \\
& =\frac{\lambda_{T, \max } \sqrt{N} \alpha_{\max }}{\left\|\mathbf{t}_{T}\right\|} \mathbf{V}_{T}\left[\left\|\mathbf{t}_{T}\right\|, 0,0, \cdots\right]^{H} \\
& =\frac{\lambda_{T, \max } \sqrt{N} \alpha_{\max }}{\left\|\mathbf{t}_{T}\right\|} \mathbf{t}_{T} .
\end{aligned}
$$

This is to say that $\mathbf{C}_{I}^{H} \mathbf{a}^{*}$ is parallel to $\mathbf{t}_{T}$ being optimal.

- Under the special channel condition, it is optimal that the RAS adjusts the interferer-RASreceiver channel vector $\mathbf{C}_{I}^{H} \mathbf{a}^{*}$ that has the same norm as the interferer-receiver channel vector $\mathbf{t}_{I}$ but in the opposite direction Its proof is given as

$$
\begin{aligned}
\mathbf{C}_{I}^{H} \mathbf{a}^{*} & =\mathbf{V}_{I} \Sigma_{I}^{H} \mathbf{U}_{I}^{H} \mathbf{U}_{T}^{H}\left[\sqrt{N} \alpha_{\max }, 0,0, \cdots\right]^{H} \\
& =-\mathbf{V}_{I}\left[\lambda_{I, 1} \alpha_{\max } \sqrt{N}, 0,0, \cdots\right]^{H} \\
& =-\mathbf{t}_{I} .
\end{aligned}
$$

- Under the special channel condition, when $N \rightarrow+\infty$, max $C(\mathbf{a})$, we have

$$
\max C(\mathbf{a}) \rightarrow \log \left(\frac{P_{T} \lambda_{T, \max }^{2}}{\sigma^{2}\left\|\mathbf{g}^{H}\right\|^{2}}\right)
$$

3) Lower Boundary: Then, we discuss the lower boundary of the maximal capacity with interference and show what kind of channel conditions that the maximal capacity can only reach the lower boundary. 
Lemma 4.3: When $\mathbf{t}_{T}^{H}=\left[\left\|\mathbf{t}_{T}\right\|, 0,0, \cdots\right] \mathbf{V}_{T}^{H}$, the lower boundary of the maximal capacity with interference is given as

$$
\begin{aligned}
& \max C(\mathbf{a}) \\
\geq & \left(1+\frac{P_{T}\left(\lambda_{T, \text { max }}^{2} \alpha_{\max }^{2} N+2 \lambda_{T, \text { max }} \alpha_{\max } \sqrt{N}\left\|\mathbf{t}_{T}^{H}\right\|+\left\|\mathbf{t}_{T}^{H}\right\|^{2}\right)}{P_{I}\left(\lambda_{I, \text { max }}^{2} \alpha_{\max }^{2} N+2 \lambda_{I, \max } \alpha_{\max } \sqrt{N}\left\|\mathbf{t}_{I}^{H}\right\|+\left\|\mathbf{t}_{I}^{H}\right\|^{2}\right)+N \alpha_{\max }^{2} \sigma^{2}\left\|\mathbf{g}^{H}\right\|^{2}+\sigma^{2}}\right),
\end{aligned}
$$

where $\lambda_{I, \max }$ is the maximal singular value of $\mathbf{C}_{I}$.

Proof: When $\mathbf{t}_{T}^{H}=\left[\left\|\mathbf{t}_{T}\right\|, 0,0, \cdots\right] \mathbf{V}_{T}^{H}$, it follows that

$$
\begin{aligned}
& \max _{\mathbf{a}} \frac{P_{T}\left\|\mathbf{t}_{T}^{H}+\mathbf{a}^{H} \mathbf{C}_{T}\right\|^{2}}{P_{I}\left\|\mathbf{t}_{I}^{H}+\mathbf{a}^{H} \mathbf{C}_{I}\right\|^{2}+\sigma^{2}\left\|\mathbf{a}^{H} \mathbf{G}\right\|^{2}+\sigma^{2}} \\
\geq & \frac{\max _{\mathbf{a}} P_{T}\left\|\mathbf{t}_{T}^{H}+\mathbf{a}^{H} \mathbf{C}_{T}\right\|^{2}}{P_{I}\left(\lambda_{I, \text { max }}^{2} \alpha_{\max }^{2} N+2 \lambda_{I, \max } \alpha_{\max } \sqrt{N}\left\|\mathbf{t}_{I}^{H}\right\|+\left\|\mathbf{t}_{I}^{H}\right\|^{2}\right)+N \alpha_{\max }^{2} \sigma^{2}\left\|\mathbf{g}^{H}\right\|^{2}+\sigma^{2}} \\
= & \frac{P_{T}\left(\lambda_{T, \max }^{2} \alpha_{\max }^{2} N+2 \lambda_{T, \text { max }} \alpha_{\max } \sqrt{N}\left\|\mathbf{t}_{T}^{H}\right\|+\left\|\mathbf{t}_{I}^{H}\right\|^{2}\right)}{P_{I}\left(\lambda_{I, \text { max }}^{2} \alpha_{\max }^{2} N+2 \lambda_{I, \max } \alpha_{\max } \sqrt{N}\left\|\mathbf{t}_{I}^{H}\right\|+\left\|\mathbf{t}_{I}^{H}\right\|^{2}\right)+N \alpha_{\max }^{2} \sigma^{2}\left\|\mathbf{g}^{H}\right\|^{2}+\sigma^{2}},
\end{aligned}
$$

where

- (32) is due to $\mathbf{a}^{H} \mathbf{C}_{I} \mathbf{C}_{I}^{H} \mathbf{a} \leq \lambda_{I, \max }^{2}\left\|\mathbf{a}^{H}\right\|^{2} \leq \lambda_{I, \max }^{2} \alpha_{\max }^{2} N$ and $\mathbf{t}_{I}^{H} \mathbf{C}_{I}^{H} \mathbf{a} \leq \lambda_{I, \max }\left\|\mathbf{t}_{I}^{H}\right\|\left\|\mathbf{a}^{H}\right\| \leq$ $\lambda_{I, \max } \alpha_{\max } \sqrt{N}$ [29];

- (33) is due to the fact that by the same ideas as the proof of Proposition 4.2, when $\mathbf{t}_{T}^{H}=$ $\left[\left\|\mathbf{t}_{T}\right\|, 0,0, \cdots\right] \mathbf{V}_{T}^{H}$, it follows $\max _{\mathbf{a}}\left\|\mathbf{t}_{T}^{H}+\mathbf{a}^{H} \mathbf{C}_{T}\right\|^{2}=\lambda_{T, \max }^{2} \alpha_{\max }^{2} N+2 \lambda_{T, \max } \alpha_{\max } \sqrt{N}\left\|\mathbf{t}_{T}^{H}\right\|+$ $\left\|\mathbf{t}_{I}^{H}\right\|^{2}$.

Then, we show a special channel condition that only the lower boundary can be reached.

Proposition 4.3: Under the channel conditions of $\mathbf{t}_{T}^{H}=\left[\left\|\mathbf{t}_{T}\right\|, 0,0, \cdots\right] \mathbf{V}_{T}^{H}, \mathbf{C}_{T}=\mathbf{C}_{I}$, and $\mathbf{t}_{T}^{H}=\mathbf{t}_{I}^{H}$, the maximal capacity with interference is given as

$\max C(\mathbf{a})$

$$
=\left(1+\frac{P_{T}\left(\lambda_{T, \max }^{2} \alpha_{\max }^{2} N+2 \lambda_{T, \max } \alpha_{\max } \sqrt{N}\left\|\mathbf{t}_{T}^{H}\right\|+\left\|\mathbf{t}_{T}^{H}\right\|^{2}\right)}{P_{I}\left(\lambda_{I, \max }^{2} \alpha_{\max }^{2} N+2 \lambda_{I, \max } \alpha_{\max } \sqrt{N}\left\|\mathbf{t}_{I}^{H}\right\|+\left\|\mathbf{t}_{I}^{H}\right\|^{2}\right)+N \alpha_{\max }^{2} \sigma^{2}\left\|\mathbf{g}^{H}\right\|^{2}+\sigma^{2}}\right),
$$


and the corresponding optimal reflection coefficients design is given by (27).

Proof: See Appendix C.

Remark 4.5: From Proposition 4.3, we obtain that under the channel conditions of $\mathbf{t}_{T}^{H}=$ $\left[\left\|\mathbf{t}_{T}\right\|, 0,0, \cdots\right] \mathbf{V}_{T}^{H}, \mathbf{C}_{T}=\mathbf{C}_{I}$, and $\mathbf{t}_{T}^{H}=\mathbf{t}_{I}^{H}$, we only achieve the lower boundary of the maximal capacity. Thus, this channel condition can be seen as the worst channel condition. The physical meaning of the channel condition is that the transmission links are the same as the interference links. Therefore, suppressing the interference channel gain by designing the reflection coefficients is equivalent to suppress the transmission channel gain.

\section{Multiple Interferers SCEnARio}

The single interferer scenario is shown in the previous sections; however, the wireless communication scenario has multiple interferers for most practical situations. Thus, it is worth studying the RAS-assisted wireless communication system in the presence of multiple interferers. When there are $K$ interferers in the wireless communication system and the $k$-th interferer owns $M_{k}$ antennas, the signal model is rewritten as

$$
y=\underbrace{\left(\mathbf{t}_{T}^{H}+\mathbf{g}^{H} \mathbf{\Phi} \mathbf{H}_{T}\right) \mathbf{x}_{T}}_{\text {Desired Signal }}+\underbrace{\sum_{k=1}^{K}\left(\mathbf{t}_{k}^{H}+\mathbf{g}^{H} \mathbf{\Phi} \mathbf{H}_{k}\right) \mathbf{x}_{k}}_{\text {Interference }}+\underbrace{\mathbf{g}^{H} \mathbf{\Phi} \mathbf{z}_{1}+z_{0}}_{\text {Noise }},
$$

where $\mathbf{x}_{k} \in \mathbb{C}^{M_{k} \times 1}$ is the interference vector from the $k$-th interferer; $\mathbf{t}_{k}^{H} \in \mathbb{C}^{1 \times M_{k}}$ is the channel vector between the $k$-th interferer and the receiver; $\mathbf{H}_{k} \in \mathbb{C}^{N \times M_{k}}$ is the channel matrix between the $k$-th interferer and the receiver. Then, $\mathbf{C}_{k}=\mathbf{G H}_{k}$ denote the cascaded channel form the $k$-th interferer to the receiver via RAS, and thus the signal model (35) is equivalent to

$$
y=\underbrace{\left(\mathbf{t}_{T}^{H}+\mathbf{a}^{H} \mathbf{C}_{T}\right) \mathbf{x}_{T}}_{\text {Desired Signal }}+\underbrace{\sum_{k=1}^{K}\left(\mathbf{t}_{k}^{H}+\mathbf{a}^{H} \mathbf{C}_{k}\right) \mathbf{x}_{k}}_{\text {Interference }}+\underbrace{\mathbf{a}^{H} \mathbf{G} \mathbf{z}_{1}+z_{0}}_{\text {Noise }} .
$$

For the new signal model (36), we design the reflection coefficients for ISR and Capacity with interference, respectively, at multiple interferers scenario.

\section{A. Reflection Coefficients Design for ISR}

For the multiple interferers scenario, ISR minimization problem (P1) is rewritten as

$$
\text { (P3) } \min _{\mathbf{a}} G(\mathbf{a})=\frac{\sum_{k=1}^{K} P_{k}\left\|\mathbf{t}_{k}^{H}+\mathbf{a}^{H} \mathbf{C}_{k}\right\|^{2}}{\sum_{k=1}^{K} P_{k}\left\|\mathbf{t}_{k}^{H}\right\|^{2}}
$$


s.t. $(10)$,

where $P_{k}$ is interference power of the $k$-th interferer. By setting $\Lambda=\left[\sqrt{P_{1}} \mathbf{C}_{1}, \cdots, \sqrt{P_{K}} \mathbf{C}_{K}\right] \in$ $\mathbb{C}^{N \times \sum_{k=1}^{K} M_{k}}$ and $\varpi^{H}=\left[\sqrt{P_{1}} \mathbf{t}_{1}^{H}, \cdots, \sqrt{P_{K}} \mathbf{t}_{K}^{H}\right] \in \mathbb{C}^{1 \times \sum_{k=1}^{K} M_{k}}$. The upper item in (37) can be expressed as

$$
G(\mathbf{a})=\frac{\sum_{k=1}^{K} P_{k}\left\|\mathbf{t}_{k}^{H}+\mathbf{a}^{H} \mathbf{C}_{k}\right\|^{2}}{\sum_{k=1}^{K} P_{k}\left\|\mathbf{t}_{k}^{H}\right\|^{2}}=\frac{\left\|\varpi^{H}+\mathbf{a}^{H} \boldsymbol{\Lambda}\right\|^{2}}{\left\|\varpi^{H}\right\|^{2}} .
$$

It follows that Problem (P3) is equivalent to

$$
(\mathrm{P} 3 *) \min _{\mathbf{a}} \frac{\left\|\varpi^{H}+\mathbf{a}^{H} \boldsymbol{\Lambda}\right\|^{2}}{\left\|\varpi^{H}\right\|^{2}}
$$

s.t. $(10)$.

It is obvious that Problem (P3*) is same to Problem (P1), and thus Algorithm 1 can be adopted to solve Problem (P1).

Remark 5.1: We discuss two special cases as follows.

- Row Full Rank Case: If $\varpi^{H}$ is row full rank, i.e. $N \geq \sum_{k=1}^{K} M_{k}=r$, and supposing constraint condition (10) is satisfied, the optimal reflection coefficient design for Problem $\left(\mathrm{P} 3^{*}\right)$ is given as

$$
\hat{\mathbf{a}}=-\Lambda\left(\Lambda^{H} \Lambda\right)^{-1} \varpi
$$

and the corresponding optimal value for Problem $\left(\mathrm{P} 1^{*}\right)$ is $\min _{\mathbf{a}} G(\mathbf{a})=0$. It indicates that when the number of reflection amplifiers is greater than the total numbers of the antennas at all interferers, the interference can be completely suppressed.

- Column Full Rank Case: If $\varpi^{H}$ is column full rank, i.e., $\sum_{k=1}^{K} M_{k} \geq N=r$, and supposing constraint condition (10) is satisfied, the optimal reflection coefficients design for Problem $(\mathrm{P} 3 *)$ is given as

$$
\hat{\mathbf{a}}=-\left(\Lambda \Lambda^{H}\right)^{-1} \Lambda \varpi
$$

Then, we define $\bar{\varpi}=\Omega^{H} \varpi=\left[\bar{\varpi}_{1}, \bar{\varpi}_{2}, \cdots, \bar{\varpi}_{M_{S}}\right]$, where $M_{S}=\sum_{k=1}^{K} M_{k}, \Omega^{H} \in \mathbb{C}^{M_{S} \times M_{s}}$ is an unitary matrix obtained by the SVD of $\Lambda$, i.e., $\Lambda=\Pi \Sigma_{S} \Omega^{H}$ and $\Sigma_{S} \in \mathbb{C}^{N \times M_{S}}$ is a rectangular matrix whose diagonal elements are non-negative real numbers and whose 
other elements are zero. By the same idea of the proof of Proposition 3.1, we can obtain the optimal value of Problem (P3) is given by

$$
\min _{\mathbf{a}} G(\mathbf{a})=\frac{\sum_{m=N+1}^{M_{S}}\left|\bar{\varpi}_{m}\right|^{2}}{\|\bar{\varpi}\|^{2}}
$$

From (42), we can observe that the minimal diversity of the total interference signals is $\left(\sum_{k=1}^{K} M_{k}-N\right)^{+}$.

\section{B. Reflection Coefficients Design for Capacity with Interference}

For the multiple interferers scenario, the capacity of RAS-assisted communication system with interference is rewritten as

$$
\begin{aligned}
& \log \left(1+\frac{P_{T}\left\|\mathbf{t}_{T}^{H}+\mathbf{a}^{H} \mathbf{C}_{T}\right\|^{2}}{\sum_{k=1}^{K} P_{k}\left\|\mathbf{t}_{k}^{H}+\mathbf{a}^{H} \mathbf{C}_{k}\right\|^{2}+\sigma^{2}\left\|\mathbf{a}^{H} \mathbf{G}\right\|^{2}+\sigma^{2}}\right) \\
= & \log \left(1+\frac{P_{T}\left\|\varpi^{H}+\mathbf{a}^{H} \mathbf{C}_{T}\right\|^{2}}{\left\|\varpi^{H}+\mathbf{a}^{H} \boldsymbol{\Lambda}\right\|^{2}+\sigma^{2}\left\|\mathbf{a}^{H} \mathbf{G}\right\|^{2}+\sigma^{2}}\right) .
\end{aligned}
$$

Thus, the capacity maximization problem for the multiple interferer scenarios is formulated as

$$
\max _{\mathbf{a}} \log \left(1+\frac{P_{T}\left\|\mathbf{t}_{T}^{H}+\mathbf{a}^{H} \mathbf{C}_{T}\right\|^{2}}{\left\|\varpi^{H}+\mathbf{a}^{H} \boldsymbol{\Lambda}\right\|^{2}+\sigma^{2}\left\|\mathbf{a}^{H} \mathbf{G}\right\|^{2}+\sigma^{2}}\right)
$$

s.t. $(10)$.

Due to the fact that the function $\log (\cdot)$ is monotonically increasing, the above problem is equivalent to Problem (P4)

$$
\text { (P4) } \max _{\mathbf{a}} \frac{P_{T}\left\|\mathbf{t}_{T}^{H}+\mathbf{a}^{H} \mathbf{C}_{T}\right\|^{2}}{\left\|\varpi^{H}+\mathbf{a}^{H} \boldsymbol{\Lambda}\right\|^{2}+\sigma^{2}\left\|\mathbf{a}^{H} \mathbf{G}\right\|^{2}+\sigma^{2}}
$$

s.t. $(10)$.

It is obvious that the Problem (P4) is similar to Problem (P2), and thus it can be solved by Algorithm 2.

Remark 5.2: Finally, we discuss the upper and lower boundaries of the maximal capacity for the multiple interference scenario and what kind of channel conditions can achieve them.

- Upper Boundary: By the same ideas as the proofs of Lemma 4.2 and Proposition 4.2, we derive that the upper boundary of capacity is also given by (26). Under the channel 
conditions of $\mathbf{t}_{T}^{H}=\left[\left\|\mathbf{t}_{T}\right\|, 0,0, \cdots\right] \mathbf{V}_{T}^{H}, \mathbf{t}_{k}^{H}=\left[\lambda_{k, 1} \alpha_{\max } \sqrt{N}, 0,0, \cdots\right] \mathbf{V}_{k}^{H}$, and $\mathbf{U}_{T}=$ $-\mathbf{U}_{k}, k=1, \cdots, K$, the upper boundary can be achieved, where $\mathbf{U}_{k}, k=1 \cdots, K$ are the unitary matrixes obtained by SVD of $\mathbf{C}_{k}=\mathbf{U}_{k} \Sigma_{k} \mathbf{V}_{k}^{H}$. The corresponding optimal reflection coefficients is $\mathbf{a}^{*}=\mathbf{U}_{T}\left[\sqrt{N} \alpha_{\max }, 0,0, \cdots\right]^{H}$.

- Lower Boundary: By the same ideas as the proofs of Lemma 4.3 and Proposition 4.3. we can prove that when $\mathbf{t}_{T}^{H}=\left[\left\|\mathbf{t}_{T}\right\|, 0,0, \cdots\right] \mathbf{V}_{T}^{H}$, the lower boundary of capacity is given by

$\max C(\mathbf{a})$

$\geq\left(1+\frac{P_{T}\left(\lambda_{T, \max }^{2} \alpha_{\max }^{2} N+2 \lambda_{T, \max } \alpha_{\max } \sqrt{N}\left\|\mathbf{t}_{T}^{H}\right\|+\left\|\mathbf{t}_{T}^{H}\right\|^{2}\right)}{\sum_{k=1}^{K} P_{k}\left(\lambda_{k, \text { max }}^{2} \alpha_{\max }^{2} N+2 \lambda_{k, \text { max }} \alpha_{\max } \sqrt{N}\left\|\mathbf{t}_{k}^{H}\right\|+\left\|\mathbf{t}_{k}^{H}\right\|^{2}\right)+N \alpha_{\max }^{2} \sigma^{2}\left\|\mathbf{g}^{H}\right\|^{2}+\sigma^{2}}\right)$.

Then, under the channel conditions of $\mathbf{C}_{T}=\mathbf{C}_{k}$ and $\mathbf{t}_{T}^{H}=\mathbf{t}_{k}^{H}, k=1, \cdots, K$, the maximal capacity can only reach the lower boundary, and the corresponding optimal reflection coefficients is also $\mathbf{a}^{*}=\mathbf{U}_{T}\left[\sqrt{N} \alpha_{\max }, 0,0, \cdots\right]^{H}$.

\section{NUMERICAL RESULtS}

In this section, we validate our analysis by simulations. The all channels are considered as Rician Fading model. The channels of the transmitter-RAS link, the transmitter-receiver link, the interferer-RAS link, the interferer-receiver link, and the RAS-receiver links are denoted as $\mathbf{H}_{T}=\sqrt{\frac{B}{B+1}} \overline{\mathbf{H}}_{T}+\sqrt{\frac{1}{B+1}} \hat{\mathbf{H}}_{T}, \mathbf{t}_{T}=\sqrt{\frac{B}{B+1}} \overline{\mathbf{H}}_{T}+\sqrt{\frac{1}{B+1}} \hat{\mathbf{H}}_{T}, \mathbf{H}_{I}=\sqrt{\frac{B}{B+1}} \overline{\mathbf{H}}_{I}+\sqrt{\frac{1}{B+1}} \hat{\mathbf{H}}_{I}$, $\mathbf{t}_{I}=\sqrt{\frac{B}{B+1}} \overline{\mathbf{t}}_{I}+\sqrt{\frac{1}{B+1}} \hat{\mathbf{t}}_{I}$, and $\mathbf{g}=\sqrt{\frac{B}{B+1}} \overline{\mathbf{g}}+\sqrt{\frac{1}{B+1}} \hat{\mathbf{g}}$, respectively, where $B$ is the Ricain factor; $\overline{\mathbf{H}}_{T}, \overline{\mathbf{t}}_{T} \overline{\mathbf{H}}_{I}, \overline{\mathbf{t}}_{I}$ are the non-line-of-sight (NLoS) components, each element of those being i.i.d. complex Gaussian distribution $\mathcal{C N}(0,1)$. Los components are expressed by the responses of the uniform linear array (ULA), which is $\Upsilon_{N}(\theta)=\left[1, e^{j 2 \pi \frac{d}{w} \sin (\theta)}, \cdots, e^{j 2 \pi \frac{d}{w}(N-1) \sin (\theta)}\right]^{H}$, where $N$ is the number of elements for ULA. Thus, the LoS components of the above channels are given as $\hat{\mathbf{H}}_{T}=\Upsilon_{N}\left(\theta_{A o A}\right) \Upsilon_{M_{T}}\left(\theta_{A o D}\right)^{H}, \hat{\mathbf{t}}_{T}=\Upsilon_{M_{T}}\left(\theta_{A o D}\right), \hat{\mathbf{H}}_{I}=\Upsilon_{N}\left(\theta_{A o A}\right) \Upsilon_{M_{I}}\left(\theta_{A o D}\right)^{H}$, $\hat{\mathbf{t}}_{I}=\Upsilon_{M_{I}}\left(\theta_{A o D}\right)$, and $\hat{\mathbf{g}}=\Upsilon_{N}\left(\theta_{A o D}\right)$, respectively, where $\theta_{A o A}$ and $\theta_{A o D}$ are angle of arrival and angle of departure, respectively. In this simulation, we set $\frac{d}{w}=1, B=1, \alpha_{\max }=3 . \theta_{A o A}$ and $\theta_{A o D}$ are randomly set within $[0,2 \pi)$. The numerical results in this section are averaged over 200 random realization.

In comparison, except for Algorithms 1 and 2, we compute the capacity with interference under the following benchmark scheme: 


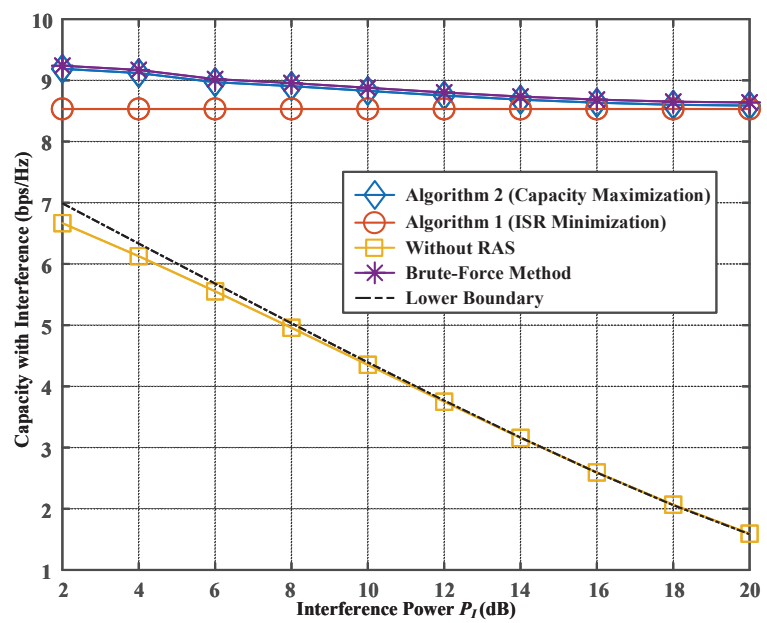

(a) Capacity with Interference

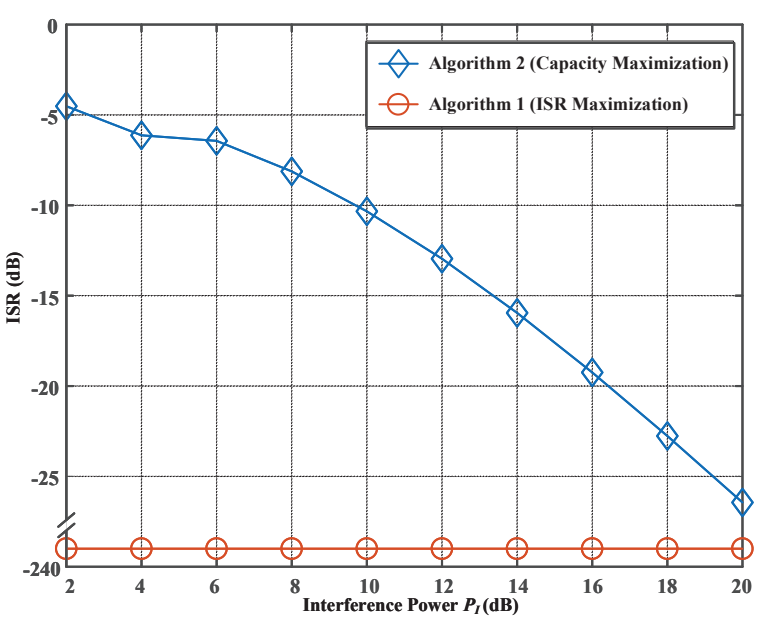

(b) ISR

Fig. 3. Capacity with Interference and ISR versus the Interference Power.

- Without RAS: We compute the capacity with interference without using RAS, which is given by

$$
\log \left(1+\frac{P_{T}\left\|\mathbf{t}_{T}\right\|^{2}}{P_{I}\left\|\mathbf{t}_{I}\right\|^{2}+\sigma^{2}}\right)
$$

- Brute-Force Method: The optimal reflection coefficients for Problem (P1) is obtained by the brute-force search.

- Lower Boundary: The lower boundary of the maximal capacity is shown in (34) for the single interferer scenario and (46) for the multiple interferer scenario.

\section{A. Effect of Interference Power}

Fig. 3 shows the two targets; (a) the capacity with interference; (b) ISR, as the functions of the interference power, respectively, where $P_{T}=20 \mathrm{~dB}, N=10$, and $M_{T}=M_{I}=10$.

From Fig. 3(a), we can observe that under Algorithm 1 (i.e., ISR minimization), the capacity remains constant with the interference power $P_{I}$ increasing. This is due to the fact that when $N=M_{I}$, the interference can be perfectly suppressed.

From Fig. 3(b), we can observe that the ISR under Algorithm 2 decreases with $P_{I}$. It indicates that with the increased $P_{I}$, in order to maximize the capacity, the RAS needs to be designed mainly for the interference suppression, which has been proved and discussed in Proposition 4.1 and Remark 4.3. Moreover, from Fig. 3(a), we observe that the capacity under Algorithm 2 (i.e., 


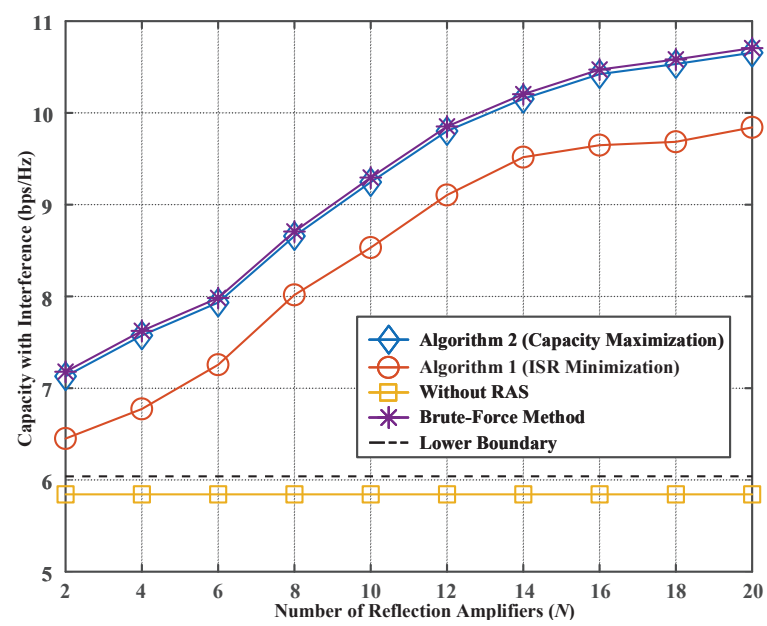

(a) Capacity with Interference

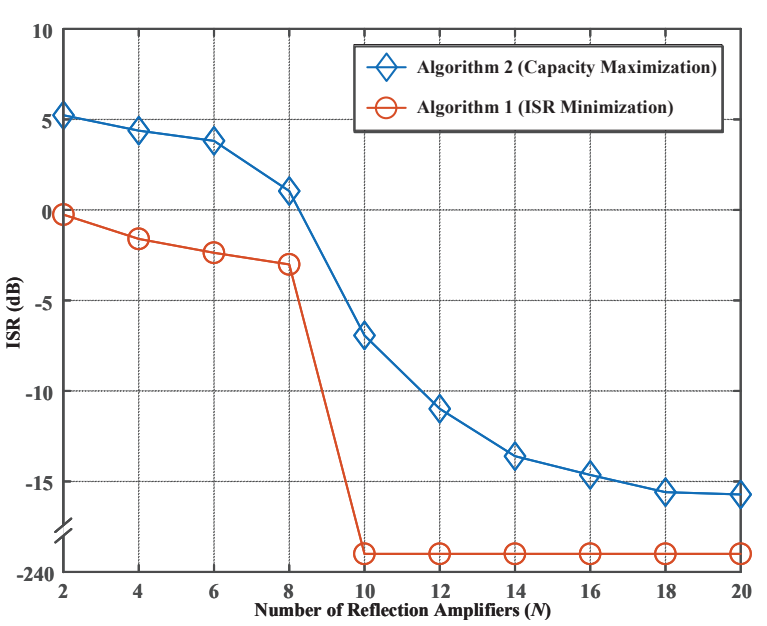

(b) ISR

Fig. 4. Capacity with Interference and ISR versus Number of Reflection Amplifiers.

capacity maximization) slightly decreases and gets close to the capacity under Algorithm 1 as $P_{I}$ increases. It implies that with the $P_{I}$ increased, the reflection coefficients design for the two optimum targets (capacity maximization and ISR minimization) become remarkably similar.

\section{B. Effect of Number of Reflection Amplifiers}

Fig. 4 represents the two targets; (a) the capacity with interference; (b) ISR, versus the number of reflection amplifiers, respectively, where $P_{T}=20 \mathrm{~dB}, P_{I}=5 \mathrm{~dB}$, and $M_{T}=M_{I}=10$. From Fig. 4(a), we observe that the capacities under Algorithms 1 and 2 both increase as the number of reflection amplifiers increases.

The increase of capacity under Algorithms 2 is because more reflection amplifiers provide more free-of-degree to enhance the transmission channel gain and weaken the interference channel gain. The increase of capacity under Algorithms 1 is due to the diversity order of the interference links decreasing with $N$.

It is worth to noticing that when $N \geq M_{I}$, the interference channel gain under Algorithm 1 has been perfectly suppressed (Please see Fig. 4(b)). The capacity under Algorithm 1 still increase with $N$ increasing. It implies that even though without the reflection coefficients design, the increased $N$ can still increases the transmission channel gain.

In addition, from Fig. 4(b), we can observe that when the number of reflection amplifiers is few, the ISR is even greater than $0 \mathrm{~dB}$ under Algorithm 2, i.e., the RAS boosts the interference 


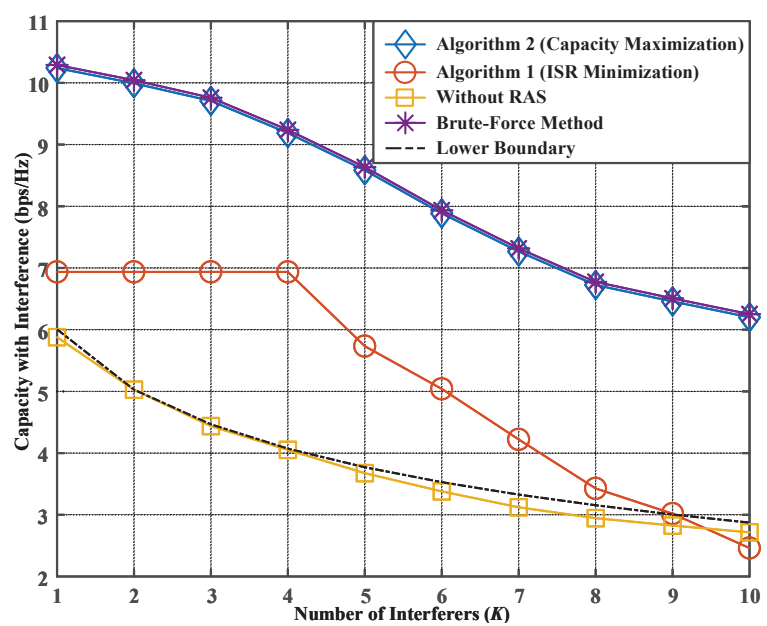

(a) Capacity with Interference

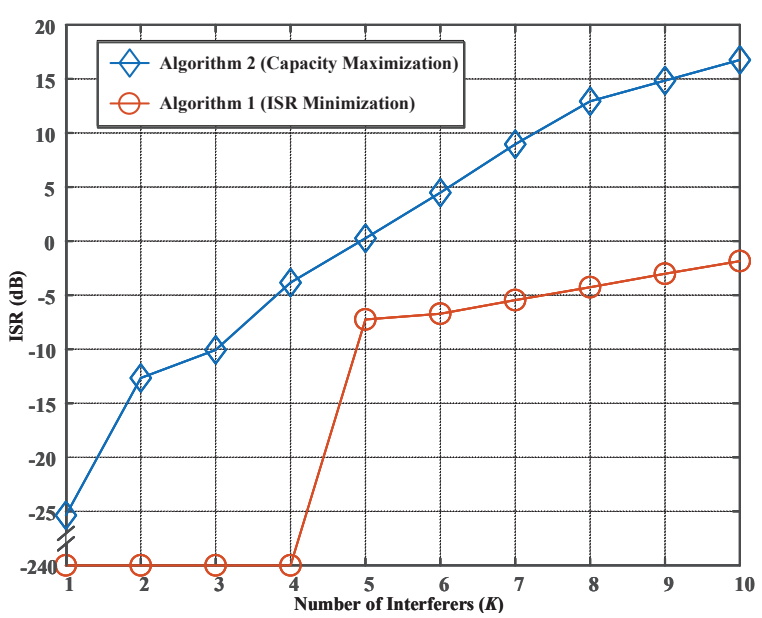

(b) ISR

Fig. 5. Capacity with Interference and ISR versus the Number of Interferers.

power. It indicates that when $N$ is few, although the RAS increasing the transmission channel gain will bring more interference, it is still beneficial for raising the capacity.

\section{Effect of Number of Interferers}

Fig. 5 represents the two targets; (a) the capacity with interference; (b) ISR, versus the number of reflection amplifiers, In Fig. 5, we plot the capacity with interference and the ISR versus the number of interferers, respectively, where $P_{T}=20 \mathrm{~dB}, P_{I}=5 \mathrm{~dB}, N=20, M_{T}=5$, and $M_{k}=5, k=1, \cdots, K$.

From Fig. 5(b), we can observe that when $N \geq \sum_{k=1}^{K} M_{k}$, the interference under Algorithm 1 is perfectly suppressed. Thus, the capacity under Algorithm 1 keeps the constant for $N \geq$ $\sum_{k=1}^{K} M_{k}$. Then, since the interference cannot be perfectly suppressed for $N<\sum_{k=1}^{K} M_{k}$, the capacity under Algorithm 1 starts to decrease with $K$ increasing.

The capacity under Algorithm 2 reduces as the number of interferers $K$ increases, and the ISR under Algorithm 1 rises as $K$ increases. It implies that the effect on increasing capacity due to the interference suppression reduces over the number of interferers. Thus, to maximize the capacity, the RAS mainly focuses on enhancing the transmission channel gain and ignores suppressing the interference channel gain. 


\section{CONCLUSIONS}

This paper proposed the RAS-based approach to suppress the co-channel interference and enhance the desired signal in MISO communication in the presence of the interferer. First, based on the proposed communication system, this paper designed the reflection coefficients to optimize the ISR. In addition, this paper proved that when the number of reflection amplifiers is greater than the number of antennas at the interferer, the interference can be perfectly suppressed in the spatial domain. Even though the number of reflection amplifiers is less than the number of antennas at the interferer, the diversity of antennas at the interferes can be counteracted by the RAS. Then, this paper proposed an iterative algorithm to obtain the optimal reflection coefficients for maximizing the capacity of RAS-assisted wireless communication. Moreover, it was concluded that the ISR optimization is almost equivalent to the capacity maximization for the strong interference case. Besides, this paper obtained the upper and lower boundaries for the maximal capacity, and showed what kind of channel conditions can achieve the two boundaries and the corresponding optimal reflection coefficients. Finally, the above results were extended to multiple interferers scenario.

\section{APPENDIX A}

\section{PROOF OF PROPOSITION 3.1}

Substituting the optimal solution for Problem $(\mathrm{P} 1 *)$ under the column full rank case given by (15) into $G(\mathbf{a})$, it follows that

$$
\begin{aligned}
& \min _{\mathbf{a}} G(\mathbf{a})=\frac{\left\|\mathbf{t}_{I}-\mathbf{C}_{I}^{H}\left(\mathbf{C}_{I} \mathbf{C}_{I}^{H}\right)^{-1} \mathbf{C}_{I} \mathbf{t}_{I}\right\|^{2}}{\left\|\mathbf{t}_{I}\right\|^{2}} \\
& =\frac{\left\|\mathbf{t}_{I}-\mathbf{V}_{I} \Sigma_{I}^{H} \mathbf{U}_{I}^{H}\left(\mathbf{U}_{I} \Sigma_{I} \mathbf{V}_{I}^{H} \mathbf{V}_{I} \Sigma_{I}^{H} \mathbf{U}_{I}^{H}\right)^{-1} \mathbf{U}_{I} \Sigma_{I} \mathbf{V}_{I}^{H} \mathbf{t}_{I}\right\|^{2}}{\left\|\mathbf{t}_{I}\right\|^{2}} \\
& =\frac{\left\|\mathbf{t}_{I}-\mathbf{V}_{I} \Sigma_{I}^{H}\left(\Sigma_{I} \Sigma_{I}^{H}\right)^{-1} \Sigma_{I} \mathbf{V}_{I}^{H} \mathbf{t}_{I}\right\|^{2}}{\left\|\mathbf{t}_{I}\right\|^{2}} \\
& =\frac{\left\|\mathbf{t}_{I}-\mathbf{V}_{I}\left[\begin{array}{cc}
\mathbf{I}_{N \times N} & \mathbf{0}_{N \times\left(M_{I}-N\right)} \\
\mathbf{0}_{\left(M_{I}-N\right) \times N} & \mathbf{0}_{\left(N-M_{I}\right)}
\end{array}\right] \mathbf{V}_{I}^{H} \mathbf{t}_{I}\right\|^{2}}{\left\|\mathbf{t}_{I}\right\|^{2}}
\end{aligned}
$$




$$
\begin{aligned}
& =\frac{\overline{\mathbf{t}}_{I}^{H}\left[\begin{array}{cc}
\mathbf{0}_{N \times N} & \mathbf{0}_{N \times\left(M_{I}-N\right)} \\
\mathbf{0}_{\left(M_{I}-N\right) \times N} & \mathbf{I}_{\left(N-M_{I}\right)}
\end{array}\right] \overline{\mathbf{t}}_{I}}{\left\|\mathbf{t}_{I}\right\|^{2}} \\
& =\frac{\sum_{m=N+1}^{M_{I}}\left|\bar{t}_{m}\right|^{2}}{\left\|\overline{\mathbf{t}}_{I}^{H}\right\|^{2}},
\end{aligned}
$$

where

- (48) is due to (15);

- (49) is due to $\mathbf{V}_{I} \mathbf{V}_{I}^{H}=\mathbf{I}_{M}$ and $\mathbf{U}_{I} \mathbf{U}_{I}^{H}=\mathbf{I}_{N}$;

- (50) is due to $\overline{\mathbf{t}}_{I}^{H}=\mathbf{t}_{I}^{H} \mathbf{V}_{I}$.

\section{APPENDIX B}

\section{PROOF OF PROPOSITION 4.2}

First, when $\mathbf{a}=\mathbf{U}_{T}\left[\sqrt{N} \alpha_{\max }, 0,0, \cdots\right]^{H}$ and $\mathbf{t}_{T}^{H}=\left[\left\|\mathbf{t}_{T}\right\|, 0,0, \cdots\right] \mathbf{V}_{T}^{H}$, we obtain

$$
\begin{aligned}
\left\|\mathbf{t}_{T}^{H}+\mathbf{a}^{H} \mathbf{C}_{T}\right\|^{2}= & \left(\mathbf{a}^{H} \mathbf{C}_{T} \mathbf{C}_{T}^{H} \mathbf{a}+2 \mathbf{t}_{T}^{H} \mathbf{C}_{T}^{H} \mathbf{a}+\left\|\mathbf{t}_{T}^{H}\right\|^{2}\right) \\
= & \left(\mathbf{a}^{H} \mathbf{U}_{T} \Sigma_{T} \Sigma_{T}^{H} \mathbf{U}_{T}^{H} \mathbf{a}+2 \mathbf{t}_{T}^{H} \mathbf{V}_{T} \Sigma_{T}^{H} \mathbf{U}_{T}^{H} \mathbf{a}+\left\|\mathbf{t}_{T}^{H}\right\|^{2}\right) \\
= & \left(\left[\sqrt{N} \alpha_{\max }, 0,0, \cdots\right]^{H} \Sigma_{T} \Sigma_{T}^{H}\left[\sqrt{N} \alpha_{\max }, 0,0, \cdots\right]\right. \\
& \left.+2\left[\left\|\mathbf{t}_{T}\right\|, 0,0, \cdots\right] \Sigma_{T}^{H}\left[\sqrt{N} \alpha_{\max }, 0,0, \cdots\right]^{H}+\left\|\mathbf{t}_{T}^{H}\right\|^{2}\right) \\
= & \lambda_{T, \max }^{2} \alpha_{\max }^{2} N+2 \lambda_{T, \max } \alpha_{\max } \sqrt{N}\left\|\mathbf{t}_{T}^{H}\right\|+\left\|\mathbf{t}_{T}^{H}\right\|^{2},
\end{aligned}
$$

where (52) is due to $\lambda_{T, 1}=\lambda_{T, \max }$.

Then, when $\mathbf{a}=\mathbf{U}_{T}\left[\sqrt{N} \alpha_{\max }, 0,0, \cdots\right]^{H}, \mathbf{U}_{T}=-\mathbf{U}_{I}$, and $\mathbf{t}_{I}^{H}=\left[\lambda_{I, 1} \alpha_{\max } \sqrt{N}, 0,0, \cdots\right] \mathbf{V}_{I}^{H}$, we obtain

$$
\begin{aligned}
\left\|\mathbf{t}_{I}^{H}+\mathbf{a}^{H} \mathbf{C}_{I}\right\|= & \left(\mathbf{a}^{H} \mathbf{U}_{I} \Sigma_{I} \Sigma_{I}^{H} \mathbf{U}_{I}^{H} \mathbf{a}+2 \mathbf{t}_{I}^{H} \mathbf{V}_{I} \Sigma_{I}^{H} \mathbf{U}_{I}^{H} \mathbf{a}+\left\|\mathbf{t}_{I}^{H}\right\|^{2}\right) \\
= & \left(\left[\sqrt{N} \alpha_{\max }, 0,0, \cdots\right]^{H} \Sigma_{I} \Sigma_{I}^{H}\left[\sqrt{N} \alpha_{\max }, 0,0, \cdots\right]\right. \\
& \left.-2\left[\lambda_{I, 1} \alpha_{\max } \sqrt{N}, 0,0, \cdots\right] \Sigma_{I}^{H}\left[\sqrt{N} \alpha_{\max }, 0,0, \cdots\right]+\left\|\mathbf{t}_{I}^{H}\right\|^{2}\right) \\
= & \lambda_{I, 1}^{2} \alpha_{\max }^{2} N-2 \lambda_{I, 1}^{2} \alpha_{\max }^{2} N+\lambda_{I, 1}^{2} \alpha_{\max }^{2} N \\
= & 0,
\end{aligned}
$$


where

- (53) is due to $\mathbf{U}_{T}=-\mathbf{U}_{I}$;

- (54) is due to $\left\|\mathbf{t}_{I}^{H}\right\|^{2}=\lambda_{I, 1}^{2} \alpha_{\max }^{2} N$.

Next, when $\mathbf{a}=\mathbf{U}_{T}\left[\sqrt{N} \alpha_{\max }, 0,0, \cdots\right]^{H}$, we can obtain

$$
\begin{aligned}
\sigma^{2}\left\|\mathbf{a}^{H} \mathbf{G}\right\|^{2} & =\sigma^{2} \mathbf{a}^{H} \mathbf{G} \mathbf{G}^{H} \mathbf{a} \\
& =\sigma^{2} \sqrt{N} \alpha_{\max } \mathbf{u}_{T, 1}^{H} \operatorname{diag}(\mathbf{g}) \operatorname{diag}\left(\mathbf{g}^{H}\right) \mathbf{u}_{T, 1} \alpha_{\max } \sqrt{N} \\
& =\sigma^{2} N^{2} \alpha_{\max }^{2} \sum_{n=1}^{N}\left|g_{n}\right|^{2} \mathbf{u}_{T, 1}^{H} \mathbf{u}_{T, 1} \\
& =\sigma^{2} N^{2} \alpha_{\max }^{2}\left\|\mathbf{g}^{\mathbf{H}}\right\|^{2},
\end{aligned}
$$

where

- (56) is due to the fact that we denote unitary matrice $\mathbf{U}_{T}=\left[\mathbf{u}_{1, T}, \cdots, \mathbf{u}_{N, T}\right]$ and thus $\mathbf{U}_{T}\left[\sqrt{N} \alpha_{\max }, 0,0, \cdots\right]^{H}=\sqrt{N} \alpha_{\max } \mathbf{u}_{1, T} ;$

- (57) is due to $\mathbf{g}=\left[g_{1}, \cdots, g_{N}\right]^{H}$;

- (58) is due to $\left\|\mathbf{g}^{\mathbf{H}}\right\|^{2} \sum_{n=1}^{N}\left|g_{n}\right|^{2}$ and $\mathbf{u}_{T, 1}^{H} \mathbf{u}_{T, 1}=1$.

Finally, based on (52), (54), (58) and (17), we obtain can (26). From Lemma 4.2, we learn (26) is the upper boundary of the maximal capacity, and it follows that $\mathbf{a}=\mathbf{U}_{T}\left[\sqrt{N} \alpha_{\max }, 0,0, \cdots\right]^{H}$ is the optimal reflection coefficients design.

\section{APPENDIX C}

\section{PROOF OF PROPOSITION 4.3}

When $\mathbf{C}_{T}^{H}=\mathbf{C}_{I}^{H}$ and $\mathbf{t}_{T}^{H}=\mathbf{t}_{I}^{H}$, it follows that

$$
\begin{aligned}
& \frac{P_{T}\left\|\mathbf{t}_{T}^{H}+\mathbf{a}^{H} \mathbf{C}_{T}\right\|^{2}}{P_{I}\left\|\mathbf{t}_{I}^{H}+\mathbf{a}^{H} \mathbf{C}_{I}\right\|^{2}+\sigma^{2}\left\|\mathbf{a}^{H} \mathbf{G}\right\|^{2}+\sigma^{2}} \\
= & \frac{P_{T}\left\|\mathbf{t}_{T}^{H}+\mathbf{a}^{H} \mathbf{C}_{T}\right\|^{2}}{P_{I}\left\|\mathbf{t}_{T}^{H}+\mathbf{a}^{H} \mathbf{C}_{T}\right\|^{2}+\sigma^{2}\left\|\mathbf{a}^{H} \mathbf{G}\right\|^{2}+\sigma^{2}} \\
= & \frac{P_{T}}{P_{I}+\frac{\sigma^{2}\left\|\mathbf{a}^{H} \mathbf{G}\right\|^{2}+\sigma^{2}}{\left\|\mathbf{t}_{T}^{H}+\mathbf{a}^{H} \mathbf{C}_{T}\right\|^{2}}} \\
\leq & \frac{P_{T}}{P_{I}+\frac{N \alpha_{\max }^{2} \sigma^{2}\left\|\mathbf{g}^{H}\right\|^{2}+\sigma^{2}}{\lambda_{T, \text { max }}^{2} \alpha_{\max }^{2} N+2 \lambda_{T, \max } \alpha_{\max } \sqrt{N}\left\|\mathbf{t}_{T}^{H}\right\|+\left\|\mathbf{t}_{I}^{H}\right\|^{2}}}
\end{aligned}
$$




$$
=\frac{P_{T}\left(\lambda_{T, \max }^{2} \alpha_{\max }^{2} N+2 \lambda_{T, \max } \alpha_{\max } \sqrt{N}\left\|\mathbf{t}_{T}^{H}\right\|+\left\|\mathbf{t}_{T}^{H}\right\|^{2}\right)}{P_{I}\left(\lambda_{I, \max }^{2} \alpha_{\max }^{2} N+2 \lambda_{I, \max } \alpha_{\max } \sqrt{N}\left\|\mathbf{t}_{I}^{H}\right\|+\left\|\mathbf{t}_{I}^{H}\right\|^{2}\right)+N \alpha_{\max }^{2} \sigma^{2}\left\|\mathbf{g}^{H}\right\|^{2}+\sigma^{2}},
$$

where (59) is due to $\mathbf{t}_{T}^{H}=\mathbf{t}_{I}^{H}$ and $\lambda_{T, \max }=\lambda_{I, \max }$ based on $\mathbf{C}_{T}^{H}=\mathbf{C}_{I}^{H}$. It follows that

$$
\begin{aligned}
& C(\mathbf{a}) \\
& \leq\left(1+\frac{P_{T}\left(\lambda_{T, \max }^{2} \alpha_{\max }^{2} N+2 \lambda_{T, \max } \alpha_{\max } \sqrt{N}\left\|\mathbf{t}_{T}^{H}\right\|+\left\|\mathbf{t}_{T}^{H}\right\|^{2}\right)}{P_{I}\left(\lambda_{I, \max }^{2} \alpha_{\max }^{2} N+2 \lambda_{I, \max } \alpha_{\max } \sqrt{N}\left\|\mathbf{t}_{I}^{H}\right\|+\left\|\mathbf{t}_{I}^{H}\right\|^{2}\right)+N \alpha_{\max }^{2} \sigma^{2}\left\|\mathbf{g}^{H}\right\|^{2}+\sigma^{2}}\right) .
\end{aligned}
$$

Based on the Lemma 4.3 and (60), we obtain (34) under the channel conditions of $\mathbf{C}_{T}=\mathbf{C}_{I}$ and $\mathbf{t}_{T}^{H}=\mathbf{t}_{I}^{H}$. From the proof of Proposition 4.2, we know when $\mathbf{t}_{T}^{H}=\left[\left\|\mathbf{t}_{T}\right\|, 0,0, \cdots\right] \mathbf{V}_{T}^{H}$ and $\mathbf{a}=\mathbf{U}_{T}\left[\sqrt{N} \alpha_{\max }, 0,0, \cdots\right]^{H}$, we can obtain (34). Thus, $\mathbf{a}=\mathbf{U}_{T}\left[\sqrt{N} \alpha_{\max }, 0,0, \cdots\right]^{H}$ is the optimal coefficients design for the special channel conditions.

\section{REFERENCES}

[1] X. Ge, S. Tu, G. Mao, C. Wang, and T. Han, "5G ultra-dense cellular networks," IEEE Wireless Commun., vol. 23, no. 1, pp. 72-79, Feb. 2016.

[2] W. Nam, D. Bai, J. Lee, and I. Kang, "Advanced interference management for $5 \mathrm{~g}$ cellular networks," IEEE Commun. Mag., vol. 52, no. 5, pp. 52-60, May 2014.

[3] S. Hong, J. Brand, J. I. Choi, M. Jain, J. Mehlman, S. Katti, and P. Levis, "Applications of self-interference cancellation in 5g and beyond," IEEE Commun. Mag., vol. 52, no. 2, pp. 114-121, Feb. 2014.

[4] S. L. Cotton, W. G. Scanlon, and B. K. Madahar, "Millimeter-wave soldier-to-soldier communications for covert battlefield operations," IEEE Commun. Mag., vol. 47, no. 10, pp. 72-81, Oct. 2009.

[5] H. Baek and J. Lim, "Design of future uav-relay tactical data link for reliable uav control and situational awareness," IEEE Commun. Mag., vol. 56, no. 10, pp. 144-150, Oct. 2018.

[6] V. Solcanu, M. Gaiceanu, and M. Solomon, "Interference challenges on board military ships," in Proc. ISEEE, May 2019, pp. 1-6.

[7] J. Chuprun, C. Bergstrom, and A. Guzek, "Advanced interference rejection and anti-jam methods for low power mobile battlefield communications," in Proc. MILCOM, vol. 2, Nov. 1997, pp. 841-846.

[8] A. T. Le, L. C. Tran, X. Huang, Y. J. Guo, and L. Hanzo, "Analog least mean square adaptive filtering for self-interference cancellation in full duplex radios," IEEE Wireless Commun., vol. 28, no. 1, pp. 12-18, Feb. 2021.

[9] M. Yilan, O. Gurbuz, and H. Ozkan, "Integrated linear and nonlinear digital cancellation for full duplex communication," IEEE Wireless Commun., vol. 28, no. 1, pp. 20-27, Feb. 2021.

[10] H. Zhao, U. De Silva, S. Pulipati, S. B. Venkatakrishnan, S. Bhardwaj, J. L. Volakis, S. Mandal, and A. Madanayake, "A broadband multistage self-interference canceller for full-duplex MIMO radios," IEEE Trans. Microw. Theory Tech., vol. 69, no. 4, pp. 2253-2266, Apr. 2021.

[11] V. R. Cadambe and S. A. Jafar, "Interference alignment and degrees of freedom of the K-user interference channel," IEEE Trans. Inf. Theory, vol. 54, no. 8, pp. 3425-3441, Aug. 2008. 
[12] K. E. Kolodziej, J. P. Doane, B. T. Perry, and J. S. Herd, "Adaptive beamforming for multi-function in-band full-duplex applications," IEEE Wireless Commun., vol. 28, no. 1, pp. 28-35, Feb. 2021.

[13] N. Zhao, X. Liu, F. R. Yu, M. Li, and V. C. M. Leung, "Communications, caching, and computing oriented small cell networks with interference alignment," IEEE Commun. Mag., vol. 54, no. 9, pp. 29-35, Sep. 2016.

[14] D. Hwang, S. S. Nam, and J. Yang, "Multi-antenna beamforming techniques in full-duplex and self-energy recycling systems: Opportunities and challenges,” IEEE Commun. Mag., vol. 55, no. 10, pp. 160-167, Oct. 2017.

[15] C. M. Yetis, J. Fanjul, J. A. Garcia-Naya, N. N. Moghadam, and H. Farhadi, "Interference alignment testbeds," IEEE Commun. Mag., vol. 55, no. 10, pp. 120-126, Oct. 2017.

[16] B. Soret, H. Wang, K. I. Pedersen, and C. Rosa, "Multicell cooperation for LTE-advanced heterogeneous network scenarios," IEEE Wireless Commun., vol. 20, no. 1, pp. 27-34, Feb. 2013.

[17] M. Rezaee and M. Guillaud, "Interference alignment with quantized grassmannian feedback in the K-user constant MIMO interference channel," IEEE Trans. Wireless Commun., vol. 15, no. 2, pp. 1456-1468, Feb. 2016.

[18] Q. Wu and R. Zhang, "Towards smart and reconfigurable environment: Intelligent reflecting surface aided wireless network," IEEE Commun. Mag., vol. 58, no. 1, pp. 106-112, Jan. 2020.

[19] A. Sikri, A. Mathur, P. Saxena, M. R. Bhatnagar, and G. Kaddoum, "Reconfigurable intelligent surface for mixed FSO-RF systems with co-channel interference,” IEEE Commun. Lett., vol. 25, no. 5, pp. 1605-1609, May 2021.

[20] B. C. Nguyen, T. M. Hoang, L. T. Dung, and T. Kim, "On performance of two-way full-duplex communication system with reconfigurable intelligent surface," IEEE Access, vol. 9, pp. 81 274-81 285, June 2021.

[21] C. Huang, S. Hu, G. C. Alexandropoulos, A. Zappone, C. Yuen, R. Zhang, M. D. Renzo, and M. Debbah, "Holographic MIMO surfaces for 6G wireless networks: Opportunities, challenges, and trends," IEEE Wireless Commun., vol. 27, no. 5, pp. 118-125, Oct. 2020.

[22] F. Amato, C. W. Peterson, B. P. Degnan, and G. D. Durgin, "Tunneling RFID tags for long-range and low-power microwave applications," IEEE J. Radio Freq. Identification, vol. 2, no. 2, pp. 93-103, June 2018.

[23] S. Khaledian, F. Farzami, H. Soury, B. Smida, and D. Erricolo, "Active two-way backscatter modulation: An analytical study," IEEE Trans. Wireless Commun., vol. 18, no. 3, pp. 1874-1886, Mar. 2019.

[24] F. Amato, H. M. Torun, and G. D. Durgin, "RFID backscattering in long-range scenarios," IEEE Trans. Wireless Commun., vol. 17, no. 4, pp. 2718-2725, Apr. 2018.

[25] R. Long, Y.-C. Liang, Y. Pei, and E. G. Larsson, "Active reconfigurable intelligent surface aided wireless communications," IEEE Trans. Wireless Commun., vol. Early Access, IEEE Trans. Wireless Commun. 2021.

[26] S. Jeon, S. U. Hwang, I. Kyung, and J. Seo, "On the distribution of MISO channel capacity in the noise and interferencelimited systems," in Proc. IEEE Veh. Technol. Conference Fall, Sept. 2009, pp. 1-5.

[27] R. Blum, "MIMO capacity with interference," IEEE J. Sel. Areas Commun., vol. 21, no. 5, pp. 793-801, June 2003.

[28] D. Tse and P. Viswanath, Fundamentals of Wireless Communication. Cambridge, UK: Cambridge university press, 2004.

[29] J. N. Franklin, Matrix theory. New York, USA: Courier Corporation, 2012.

[30] S. Boyd and L. Vandenberghe, Convex optimization. Cambridge, UK: Cambridge university press, 2004.

[31] T. Lipp and S. Boyd, "Variations and extension of the convex-concave procedure," Optim. Eng., vol. 17, no. 2, pp. 263-287, Nov. 2016.

[32] S. Schaible and T. Ibaraki, "Fractional programming," EUR. J. OPER. RES., vol. 12, no. 4, pp. 325-338, Sep. 1983. 